BNL-114364-2017-JA

\title{
Spontaneous breaking of rotational symmetry in copper oxide superconductors
}

J. Wu

Submitted to Nature

July 2017

Condensed Matter Physics and Materials Science Department

Brookhaven National Laboratory

\author{
U.S. Department of Energy \\ USDOE Office of Science (SC), \\ Basic Energy Sciences (BES) (SC-22)
}




\section{DISCLAIMER}

This report was prepared as an account of work sponsored by an agency of the United States Government. Neither the United States Government nor any agency thereof, nor any of their employees, nor any of their contractors, subcontractors, or their employees, makes any warranty, express or implied, or assumes any legal liability or responsibility for the accuracy, completeness, or any third party's use or the results of such use of any information, apparatus, product, or process disclosed, or represents that its use would not infringe privately owned rights. Reference herein to any specific commercial product, process, or service by trade name, trademark, manufacturer, or otherwise, does not necessarily constitute or imply its endorsement, recommendation, or favoring by the United States Government or any agency thereof or its contractors or subcontractors. The views and opinions of authors expressed herein do not necessarily state or reflect those of the United States Government or any agency thereof. 


\section{Spontaneous breaking of rotational symmetry in copper oxide su-}

\section{perconductors}

J. Wu ${ }^{1}$, A. T. Bollinger ${ }^{1}$, X. He ${ }^{1,2}$ and I. Božović ${ }^{1,2}$

${ }^{1}$ Brookhaven National Laboratory, Upton, New York 11973-5000, USA

${ }^{2}$ Yale University, Applied Physics Department, New Haven CT 06520, USA

A vivid debate is ongoing about the origin of high-temperature superconductivity in copper-oxides and the nature of the 'normal' state above the critical temperature $\left(T_{c}\right){ }^{1-3}$ In underdoped copper-oxides, this state hosts a pseudo-gap and other anomalous features. On the overdoped side, the standard Bardeen-Cooper-Schrieffer description fails dramatically ${ }^{4}$, challenging the idea that the normal state is a simple Fermi liquid. Here, we report that in single-crystal $\mathrm{La}_{2-x} \mathrm{Sr}_{x} \mathrm{CuO}_{4}$ films a spontaneous voltage $\left(V_{T}\right)$ develops transverse to the electrical current. We have studied the dependence of $V_{T}$ on the probe current, temperature, in-plane device orientation, and doping. The effect is intrinsic, substantial, robust and present in a broad temperature/doping range. If the current direction is rotated in-plane by an angle $\phi, V_{T}$ oscillates as $\sin (2 \phi)$, breaking the fourfold-rotation symmetry. The amplitude of oscillations is strongly peaked near $T_{c}$, and it decreases with doping. We show that these are manifestations of in-plane anisotropy in the electronic transport, even though the films are very thin and epitaxially constrained to be tetragonal. Its origin is purely electronic - the so-called 'electronic nematicity'. The big surprise is that the director is not aligned to the crystal axes, unless a substantial orthorhombic distortion is imposed. These findings are quite unusual. The fact that this concurs with the highest-temperature superconductivity may not be a mere coincidence. 
A transverse voltage can emerge in the absence of an external magnetic field due to the anomalous Hall effect, e.g., because of spontaneous magnetization in ferromagnets, canting of spins in antiferromagnets, side hops or skew scattering from magnetic impurities, or due to topological effects (Berry curvature). ${ }^{5}$ A zero-field transverse voltage was observed in some copper-oxides close to $T_{c}$, and ascribed to vortex guiding and sample inhomogeneity ${ }^{6}$, quite likely to be present in those polycrystalline samples (see Methods Section I). In underdoped copper-oxides, the fourfold rotational symmetry breaking was observed in (magneto)transport ${ }^{7-9}$, Nernst effect $^{10-12}$, scanning tunneling microscopy ${ }^{13,14}$, Raman spectroscopy ${ }^{15}$, $\mathrm{THz}$ polarimetry ${ }^{16}$ and thermal conductivity ${ }^{17}$ studies. While these findings are closely related to ours, the difference is that we observe symmetry breaking, revealed via a non-zero $V_{T}$, in a large portion of the hole densitytemperature $(p-T)$ phase diagram, including the regions where no signs are observed of the pseudogap or of superconducting vortices. Another important difference is that in our (essentially tetragonal) films the high(er) conductivity direction is not aligned with the crystal axes and varies with doping and temperature, while in (orthorhombic) underdoped bulk crystals it is pinned to the crystal structure.

For this study, we have chosen $\mathrm{La}_{2-x} \mathrm{Sr}_{x} \mathrm{CuO}_{4}$ (LSCO), the simplest high- $T_{c}$ copper-oxide that can be doped all the way from an antiferromagnetic insulator to an overdoped non-superconducting metal. Single-crystal films of LSCO were deposited on tetragonal $\mathrm{LaSrAlO}_{4}$ (LSAO) substrates using atomic-layer-by-layer molecular-beam epitaxy. In Methods and Extended Data we provide detailed information on synthesis, characterization, lithography, transport measurements, and various tests we performed to rule out any conceivable experimental artifacts. The raw data used to generate the plots are available online.

In Fig. 1a, a sketch is shown of a typical device we employ to study the electrical transport properties. The current flows from the contact 1 to 2 in the $x$-direction, $\boldsymbol{j}=\boldsymbol{j}_{12}=j \boldsymbol{e}_{\boldsymbol{x}}$, and the transverse voltage is measured along the $y$-axis, e.g., between 3 and 4 . Our device has three pairs of transverse contacts, so we define $V_{T} \equiv\left(V_{34}+V_{56}+V_{78}\right) / 3$. Other than this averaging, we present all the data as measured and without any processing. To factor out the current and the geometry, we introduce the transverse resistivity $\rho_{T} \equiv\left(V_{T} / I_{x}\right) d$, where $I_{x} \equiv I_{12}$ is the probe current and $d$ is the film thickness. 
In Fig. 1b we show the temperature dependence of $\rho_{T}$ measured in an underdoped LSCO film with the hole density (per formula unit) $p=0.10$. The inset shows the longitudinal resistivity $\rho$ of the same sample. Figs. 1c and 1d show the analogous data for an optimally doped $(p=0.16)$ and an overdoped ( $p=0.18)$ LSCO film, respectively. Note that $\rho_{T}$ and $\rho$ have different $T$ dependences - for example, in the $p=0.10$ sample, in the interval $30 \mathrm{~K}<T<80 \mathrm{~K} \rho_{T}(T)$ decreases while $\rho(T)$ increases. For all doping levels, $\rho_{T}(T)$ shows a pronounced peak near the superconducting transition. While near $T_{c}$ the slope of $\rho(T)$ also shows a peak, $\rho_{T}(T)$ and $d \rho(T) / d T$ are not proportional.

To study the dependence of $\rho_{T}$ on the azimuth angle $\phi$ between the direction of electrical current and the crystallographic [100] direction, we have patterned the films as shown in Fig. 2a. The 'sunbeam' pattern consists of 36 Hall bars, with $\Delta \phi=10^{\circ}$ angle between the successive bars. As illustrated in Fig. 1, each Hall bar has three pairs of transverse voltage contacts. Figs. 2b-2d show that $\rho_{T}(\phi)$ is oscillating with the period of $180^{\circ}$, at every doping, both near $T_{c}$ and at the room temperature. We saw this at every other temperature and doping in thousands of devices that we have tested (see Methods Section I), as well. Note that not only the magnitude is oscillating, but also the sign of $\rho_{T}(\phi)$ is switching. Each $\rho_{T}(\phi)$ data set can be fit to a simple expression $\rho_{T}(\phi)=$ $\rho_{T}^{0} \sin [2(\phi-\alpha)]$, with two open parameters, the amplitude $\rho_{T}^{0}$ and the phase offset $\alpha$. In contrast, as seen in the bottom three panels in Figs. $2 b-2 d$, the longitudinal resistivity $\rho$ shows only small (few percent) variations that appear to be random and could originate from imperfections in the film (e.g., due to polishing defects in the substrate) or in the devices (as the result of the lithography).

The foremost question about the symmetry breaking reported here is its microscopic origin. Rotation symmetry can be broken by an unidirectional charge density wave (CDW), but no signs of CDW, static or dynamic, are seen in our LSCO films except at low doping and temperature (and even there detectable only on picosecond time scale). ${ }^{18}$ Orbital antiferromagnetism, $d$-density waves, and loop currents are predicted to occur only below the pseudo-gap temperature. A transverse voltage could originate from a peculiar topology of electronic band structure; however, a trivial Berry phase, $0 \bmod (2 \pi)$, has been observed in several hole-doped copper-oxides. ${ }^{19}$

Instead, we propose that the phenomenon reported here is a manifestation of an orthorhombic anisotropy of electron transport, so far undetected in optimally doped and overdoped copper ox- 
ides. This assumption accounts well for all of our observations. Moreover, within this scenario we predict (see Methods Section G) that the longitudinal resistivity $\rho(\phi)$ must show oscillations similar to those of $\rho_{T}(\phi)$, with the same angular period and amplitude, but phase-shifted by $\Delta \phi=$ $\pi / 4$, and superposed on the background of average resistivity, $\bar{\rho}$. (This is in contrast to $\rho_{T}(\phi)$, which is free of such a background, so that oscillations are easily discerned.) This effect is difficult to detect at high doping and temperature, because in that region $\rho_{T} / \bar{\rho}<1 \%$ and hence the amplitude of oscillations is small and comparable to the typical random sample-to-sample variations in $\bar{\rho}$. The $\rho_{T} / \bar{\rho}$ ratio becomes larger near $T_{c}$ but there the peak in $\rho_{T}$ is so sharp that even small variations in $T_{c}$ affect the measured value of $\rho_{T}$ significantly. Hence, the best place to look for this is in very underdoped samples where there is no superconductivity while the $\rho_{T} / \bar{\rho}$ ratio is large enough. As an example, in Fig. 3a we show $\rho_{T}(\phi)$ and $\rho(\phi)$ data for a strongly underdoped LSCO with $p=0.04$. Indeed, here we clearly see the oscillatory angular dependence not only of $\rho_{T}(\phi)$ but also of $\rho(\phi)$. Moreover, the period, amplitude, and phase of oscillations are just as predicted, $\rho=\bar{\rho}+\rho_{T}^{0} \cos [2(\phi-\alpha)]$. This proves that the orthorhombic anisotropy of the electron fluid is intrinsic, ruling out the possibility that the observed transverse voltage is due to contact misalignment or random sample inhomogeneity.

In-plane anisotropy of the electron transport has been anticipated theoretically and dubbed 'electronic nematicity'. ${ }^{20-30}$ [Different authors sometimes impart to 'nematicity' different meanings derived from particular theoretical models; here we use it just as an abbreviation for 'the electron fluid spontaneously breaking the crystal symmetry'.] An important question is whether the 'nematicity' reported here can legitimately be called 'electronic', hinting that it does not originate from lattice distortion. To address this question, we explored the doping dependence of both $\rho_{T}{ }^{0}$ and $\alpha$. In Fig. 4a, we show an example of the polar-coordinate plot of $\rho_{T}(\phi)$. It resembles the "cloverleaf" (d-wave symmetry) plot of the superconducting order parameter, in the momentum space, characteristic of high- $T_{c}$ copper-oxides; however, $\rho_{T}(\phi)$ shows this symmetry in the real space. The pattern reveals broken symmetry with respect to both $\hat{\mathrm{C}}_{4}$ and $\hat{\sigma}_{\mathrm{y}}$ (the mirror reflection in $x z$ plane). In Fig. $4 \mathrm{~b}$, we show that the same cloverleaf $\rho_{T}(\phi)$ dependence is seen at every doping, including in extremely underdoped films ( $p=0.02$ and $p=0.04)$ that are not superconducting. Both the amplitude $\rho_{T}^{0}$ and the offset angle $\alpha$ show systematic and strong dependences on the doped hole density $p$. As shown in Fig. $4 \mathrm{c}, \rho_{T}^{0}$ decreases fast with $p$ (roughly as $1 / p^{2}$ ) at both $T=T_{c}$ or $T=295 \mathrm{~K}$. In contrast, the longitudinal resistivity $\rho$ decreases with $p$ more gradually. 
Fig. $4 \mathrm{~d}$ shows that the $\alpha(p)$ dependence measured at room temperature is approximately linear (more details in Method Section H). In Fig. 4e, we show the doping and temperature dependence of the 'nematicity' $N \equiv \rho_{T} / \bar{\rho}=\left|\left(\rho_{\mathrm{a}}-\rho_{\mathrm{b}}\right)\right| /\left(\rho_{\mathrm{a}}+\rho_{\mathrm{b}}\right)$, as a color plot.

Three observations testify that the observed anisotropy is intrinsic to the electron fluid while the coupling to the crystal lattice is at best of secondary importance. First, the 'nematic director' (the unit vector pointing along the highest longitudinal resistivity direction, i.e., at angle $\alpha$ ) is in general not aligned with the lattice (i.e., not parallel to [100] or [010] axes, or to [110] and [11̄0] diagonals). Second, for some doping levels, the director orientation changes significantly with temperature. Third, the magnitude of nematicity in our thin films that are essentially tetragonal (Methods Section F) is equal to or even larger than in bulk crystals of the same composition that show a substantial $(\sim 1.5 \%)$ orthorhombic distortion ${ }^{8}$. However, the director orientations are very different - e.g., in bulk crystals with $p=0.02$ and $p=0.04$ at $T=295 \mathrm{~K}$ it points along the nodal direction $\left(\alpha=45^{\circ}\right.$ ), while we observe $\alpha=74^{0}$ for $p=0.02$ and $\alpha=61^{0}$ for $p=0.04$. It appears that even a substantial orthorhombic distortion merely rotates the electronic nematic axis, pinning it to the crystal structure.

To explore what controls the director orientation, we have performed several additional experiments depicted in the Extended Data. A small but ubiquitous miscut of the substrate with respect to the ideal orientation perpendicular to the [001] direction generates surface steps and terraces in every film, but we show that $\alpha$ does not depend on their orientation. Similarly, we show that moderately strong magnetic fields have essentially no effect on $\alpha$. The only extrinsic factor that we have established so far to affect $\alpha$ is the lattice distortion. We have used orthorhombic $\mathrm{NdGaO}_{3}$ (NGO) substrates and synthesized on them thin LSCO films that are epitaxially constrained to be substantially $(\sim 1 \%)$ orthorhombic, as we verified by X-ray diffraction. In such films, we also observe a cloverleaf $\rho_{T}(\phi)$ dependence, but the director is indeed pinned to one of the crystal axes at all temperatures.

So far, sightings of electronic nematicity have been confined to a relatively small portion of the phase diagram. Here we show that by a well-chosen, background-free probe (angle-resolved transverse resistivity, ARTR), nematicity is detectable at every doping and up to the room temperature. This spontaneous breaking of rotational symmetry is indeed unusual for an ordinary Fermi Liquid, posing a new challenge to the theory of high- $T_{c}$ superconductivity, in cuprates and 
beyond. ${ }^{29,30}$ It also opens the path to new experiments: if the electronic nematic order parameter detaches from the rotational symmetry of the lattice, according to a universal mechanism discussed in ref. 30, deep in the nematic superconductor a (nearly) massless, propagating rotational Goldstone mode should be present and could be detected using electromagnetic probes ${ }^{30}$.

\section{$\underline{\text { References }}$}

1. Lee, P. A., Nagaosa, N. \& Wen, X. G. Doping a Mott insulator: Physics of high-temperature superconductivity. Rev. Mod. Phys. 78, 17-85 (2006).

2. Zaanen, J., Chakravarty, S., Senthil, T., Anderson, P. W., Lee, P., Schmalian, J., Imada, M., Pines, D., Randeria, M., Varma, C., Vojta, M. \& Rice, M. Towards a complete theory of high $T_{c}$. Nature Phys. 2, 138-143 (2006).

3. Keimer, B., Kivelson, S. A., Norman, M. R., Uchida, S. \& Zaanen, J. From quantum matter to hightemperature superconductivity in copper oxides. Nature 518, 179-186 (2015).

4. Božović, I., He, X., Wu, J. \& Bollinger, A. T. Dependence of the critical temperature in overdoped copper oxides on superfluid density. Nature 536, 309-311 (2016).

5. Nagaosa, N., Sinova, J., Onoda, S., MacDonald, A. H. \& Ong, N. P. Anomalous Hall effect. Rev. Mod. Phys. 82, 1539-1592 (2010).

6. Matsuda, Y., et al. Hall anomaly in the vortex state of $\mathrm{La}_{2-x} \mathrm{Sr}_{x} \mathrm{CuO}_{4}$. Phys. Rev. B 52, 15749 (1995).

7. Ando, Y., Lavrov, A. N. \& Segawa, K. Magnetoresistance anomalies in antiferromagnetic $\mathrm{YBa}_{2} \mathrm{Cu}_{3} \mathrm{O}_{6+\times}$ : fingerprints of charged stripes. Phys. Rev. Lett. 83, 2813-2816 (1999).

8. Ando, Y., Segawa, K., Komiya, S. \& Lavrov, A. N. Electrical resistivity anisotropy from selforganized one dimensionality in high-temperature superconductors. Phys. Rev. Lett. 88, 137005 (2002).

9. Ando, Y., Lavrov, A. N. \& Komya, S. Anisotropic magnetoresistance in lightly doped $\mathrm{La}_{2-\mathrm{x}} \mathrm{Sr}_{\mathrm{x}} \mathrm{CuO}_{4}$ : Impact of antiphase domain boundaries on the electron transport. Phys. Rev. Lett. 90, 247003 (2003).

10. Daou, R. et al. Broken rotational symmetry in the pseudogap phase of a high- $T_{c}$ superconductor. Nature 463, 519-522 (2010).

11. Cyr-Choinière, O., et al. Two types of nematicity in the phase diagram of the cuprate superconductor $\mathrm{YBa}_{2} \mathrm{Cu}_{3} \mathrm{O}_{\mathrm{y}}$. Phys. Rev. B 92, 224502 (2015).

12. Li, L., Alidoust, N., Tranquada, J. M., Gu, G. D. \& Ong, N. P. Unusual Nernst effect suggesting timereversal violation in the striped cuprate superconductor $\mathrm{La}_{2-\mathrm{x}} \mathrm{Ba}_{\mathrm{x}} \mathrm{CuO}_{4}$. Phys. Rev. Lett. 107, 277001 (2011).

13. Lawler, M. J. et al. Intra-unit-cell electronic nematicity of the high- $\mathrm{T}_{\mathrm{c}}$ copper-oxide pseudogap states. Nature 466, 347-351 (2010).

14. Fujita, K. et al. Simultaneous transitions in cuprate momentum-space topology and electronic symmetry breaking. Science 344, 612-616 (2014).

15. Tassini, L. et al. Dynamical properties of charged stripes in $\mathrm{La}_{2-x} \mathrm{Sr}_{x} \mathrm{CuO}_{4}$. Phys. Rev. Lett. 95, 117002 (2005).

16. Lubashevsky, Y., Pan, L. D., Kirzhner, T., Koren, G. \& Armitage, N. P. Optical birefringence and dichroism of cuprate superconductors in the THz regime. Phys. Rev. Lett. 112, 147001 (2014).

17. Zhang, J.-C. et al. Anomalous thermal diffusivity in underdoped $\mathrm{YBa}_{2} \mathrm{Cu}_{3} \mathrm{O}_{6+x}$. Proc. Natl. Acad. Sci. USA 114, 5378-5383 (2017). 
18. Torchinsky, D. H., Mahmood, F., Bollinger, A. T., Božović, I. \& N. Gedik. Fluctuating charge density waves in a cuprate superconductor. Nature Mater. 12, 387-391 (2013).

19. Doiron-Leyraud, N., Szkopek, T., Pereg-Barnea, T., Proust, C. \& Gervais, G. Berry phase in cuprate superconductors. Phys. Rev. B 91, 245136 (2015).

20. Zaanen, J. \& Gunnarsson, O. Charged magnetic domain lines and the magnetism of high- $\mathrm{T}_{\mathrm{c}}$ oxides. Phys. Rev. B 40, 7391-7394 (1989).

21. Zaanen, J. \& Oles, A. M. Striped phase in the cuprates as a semiclassical phenomenon. Annalen der Physik 5, 224-246 (1996).

22. Kivelson, S. A., Fradkin, E. \& Emery, V. J. Electronic liquid-crystal phases of a doped Mott insulator. Nature 393, 550-553 (1998).

23. Oganesyan, V., Kivelson S. A. \& Fradkin, E. Quantum theory of a nematic Fermi fluid. Phys. Rev. B 64, 195109 (2001).

24. Hinkov, V. et al. Electronic liquid crystal state in the high-temperature superconductor $\mathrm{YBa}_{2} \mathrm{Cu}_{3} \mathrm{O}_{6.45}$. Science 319, 597-600 (2008).

25. Fradkin, E., Kivelson, S. A., Lawler, M. J., Eisenstein, J. P. \& Mackenzie, A. P. Nematic Fermi fluids in condensed matter physics. Ann. Rev. Cond. Mat. Phys. 1, 153-178 (2010).

26. Carlson, E. W. \& Dahmen, K. A. Using disorder to detect locally ordered electron nematics via hysteresis. Nature Commun. 2, 379 (2011).

27. Mesaros, A. et al. Topological defects coupling smectic modulations to intra-unit-cell nematicity in cuprates. Science 333, 426-430 (2011).

28. Phillabaum, B. V., Carlson, E. W. \& Dahmen, K. A. Spatial complexity due to bulk electronic nematicity in a superconducting underdoped cuprate. Nature Commun. 3, 915 (2011).

29. Fernandes, R. M., Chubukov, A. V. \& Schmalian, J. What drives nematic order in iron-based superconductors? Nature Phys. 10, 97-104 (2014).

30. Beekman, A. J. et al. Dual gauge field theory of quantum liquid crystals in two dimensions. Phys. Rep. 683, 1-110 (2017). 


\section{Acknowledgements}

We acknowledge valuable discussions with Z. Radović, J. Zaanen, S. A. Kivelson, A. Gozar, I. Drozdov, P. N. Armitage and J. C. Davis. The research was done at Brookhaven and was supported by the U.S. Department of Energy, Basic Energy Sciences, Materials Sciences and Engineering Division. X.H. is supported by the Gordon and Betty Moore Foundation's EPiQS Initiative through Grant GBMF4410.

\section{Author contributions}

The films were synthesized and characterized by I.B. and X.H., the lithography was done by A.T.B., the transport measurements were done by J.W., and the interpretation put forward by I.B.

\section{Author Information}

Reprints and permissions information is available at www.nature.com/reprints. The authors declare no competing financial interests. Readers are welcome to comment on the online version of this article at www.nature.com/nature. Correspondence and requests for materials should be addressed to I.B. (bozovic@bnl.gov). 
Figures
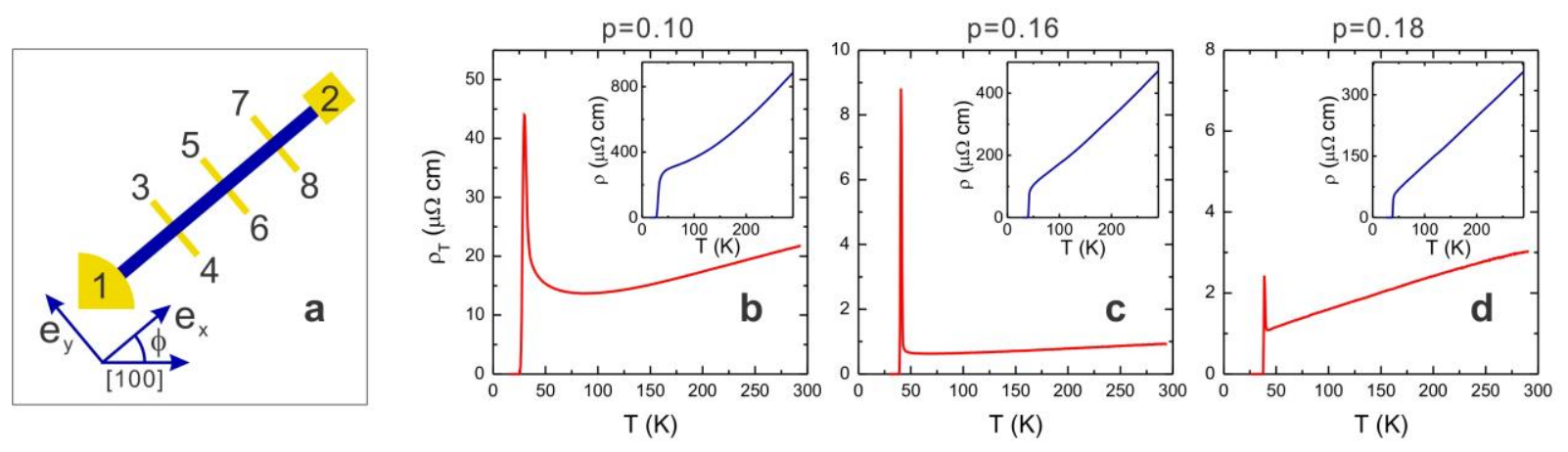

Figure 1 Temperature dependence of the longitudinal and the transverse resistivity.

a, A schematic drawing of the device used in this study. The probe current runs from the contact 1 to 2 ; the transverse voltage is measured between the contact pairs $(3,4),(5,6)$ or $(7,8)$, respectively, and $\phi$ is the angle between the current direction and the crystallographic [100] axis. The distance between the contacts 3 and 5 (or 5 and 7) is $300 \mu \mathrm{m}$; the strip width is $100 \mu \mathrm{m}$, and the width of the voltage contacts is $10 \mu \mathrm{m}$. b, Main panel: the temperature dependence of the transverse resistivity, $\rho_{T}(T)$, in an underdoped $(p=0.10)$ LSCO sample. Inset: the longitudinal resistivity $\rho(T)$ measured in the same device. c, The same, for an optimally doped ( $p=0.16)$ LSCO sample. d, The same, for an overdoped $(p=0.18)$ LSCO sample. At every doping, $\rho_{T}(T)$ shows a peak near $T_{c}$, while at higher temperatures it grows slowly and smoothly. 

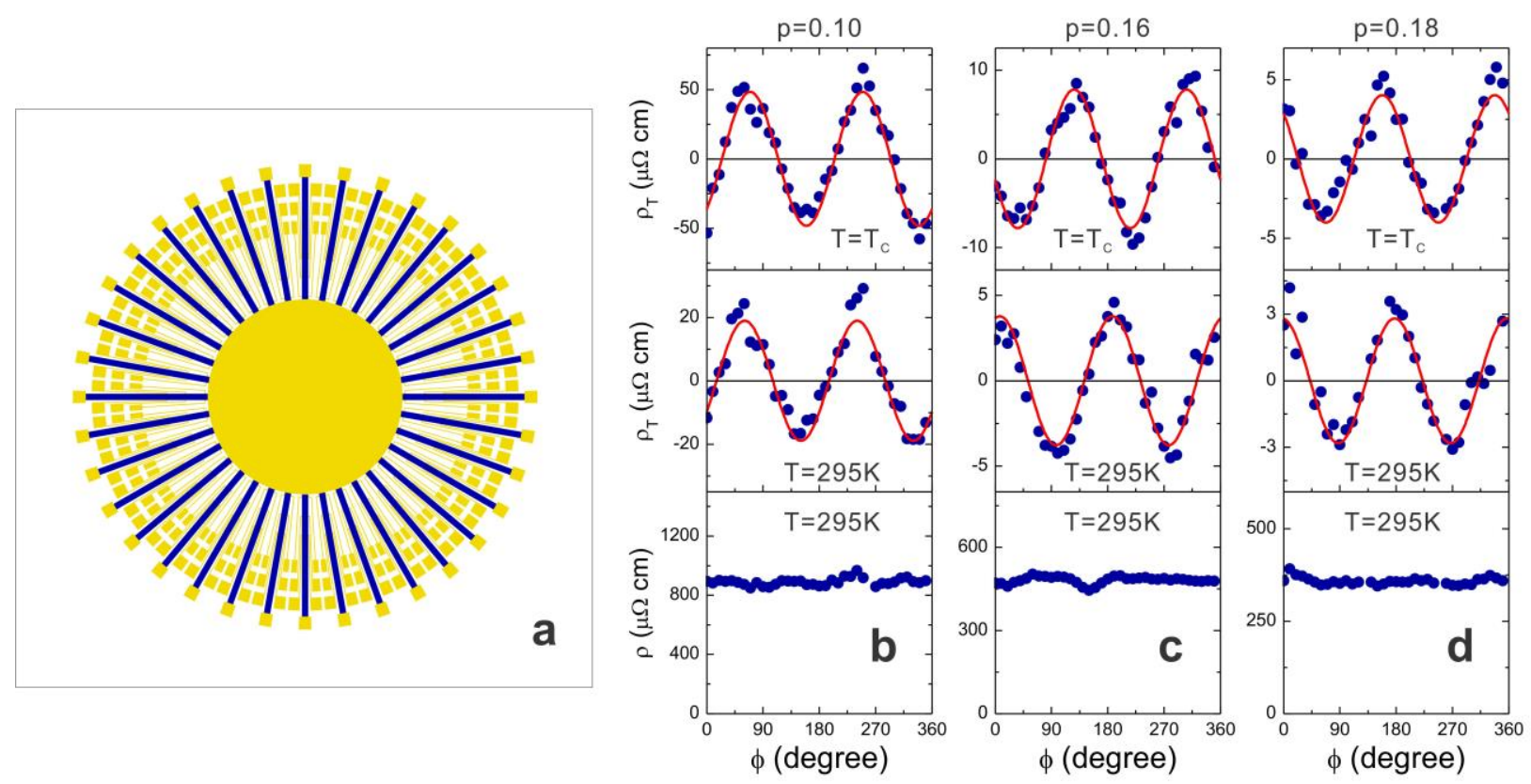

Figure 2 Angular dependence of $\rho_{T}$. a, The 'sunbeam' lithography pattern used for device fabrication contains 36 Hall bars, each with 3 pairs of transverse contacts (as sketched in Fig. 1a). The angle between two successive bars is $10^{\circ}$. $\mathbf{b}$, The $\rho_{T}(\phi)$ dependence in an underdoped $(p$ $=0.10) \mathrm{LSCO}$ film, measured at $T_{c}$-midpoint (top panel, solid blue dots) and at $T=295 \mathrm{~K}$ (middle panel). The red lines are fits to $\rho_{T}(\phi)=\rho_{T}{ }^{0} \sin [2(\phi-\alpha)]$. Bottom panel: in contrast to unmistakable oscillations in $\rho_{T}(\phi)$, the longitudinal resistivity $\rho(\phi)$ measured at $T=295 \mathrm{~K}$ shows only small (few percent) pixel-to-pixel variations that appear random. $\mathbf{c}$, The same, for an optimally doped $(p=0.16)$ LSCO sample. $\mathbf{d}$, The same, for an overdoped $(p=0.18)$ LSCO sample. 

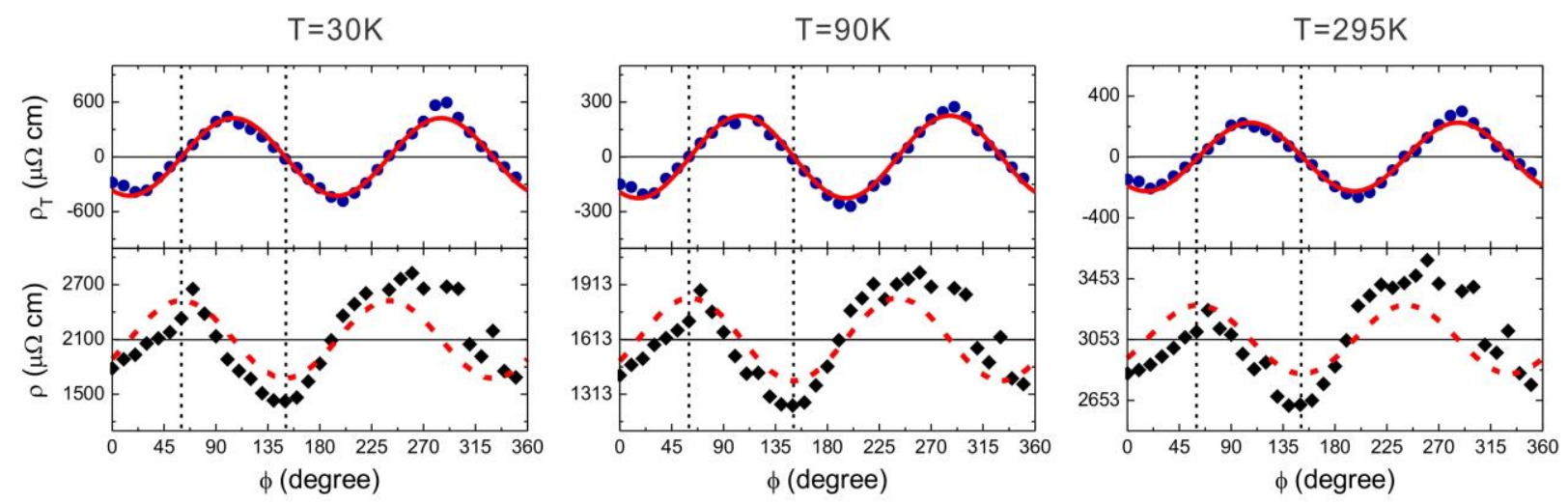

Figure 3 Relation of angular dependences of $\rho_{T}$ and $\rho$ in an underdoped $(p=0.04)$ LSCO

film. Upper panels, left to right: blue dots, the measured values of $\rho_{T}(\phi)$ at three temperatures, $T$ $=30 \mathrm{~K}, 90 \mathrm{~K}$ and $295 \mathrm{~K}$; solid red lines: fits to $\rho_{T}(\phi)=\rho_{T}{ }^{0} \sin [2(\phi-\alpha)]$ with $\rho_{T}{ }^{0}=424,226$ and $225 \mu \Omega \mathrm{cm}$ and $\alpha=60^{\circ}, 60.9^{\circ}$ and $61.7^{\circ}$, respectively. Lower panels: black diamonds, the measured values of $\rho(\phi)$; dashed red lines, the same curves as the solid red lines in the corresponding upper panels, except shifted left by $45^{\circ}$ and upward by $\bar{\rho}$, the angle-averaged resistivity at the corresponding temperature. 

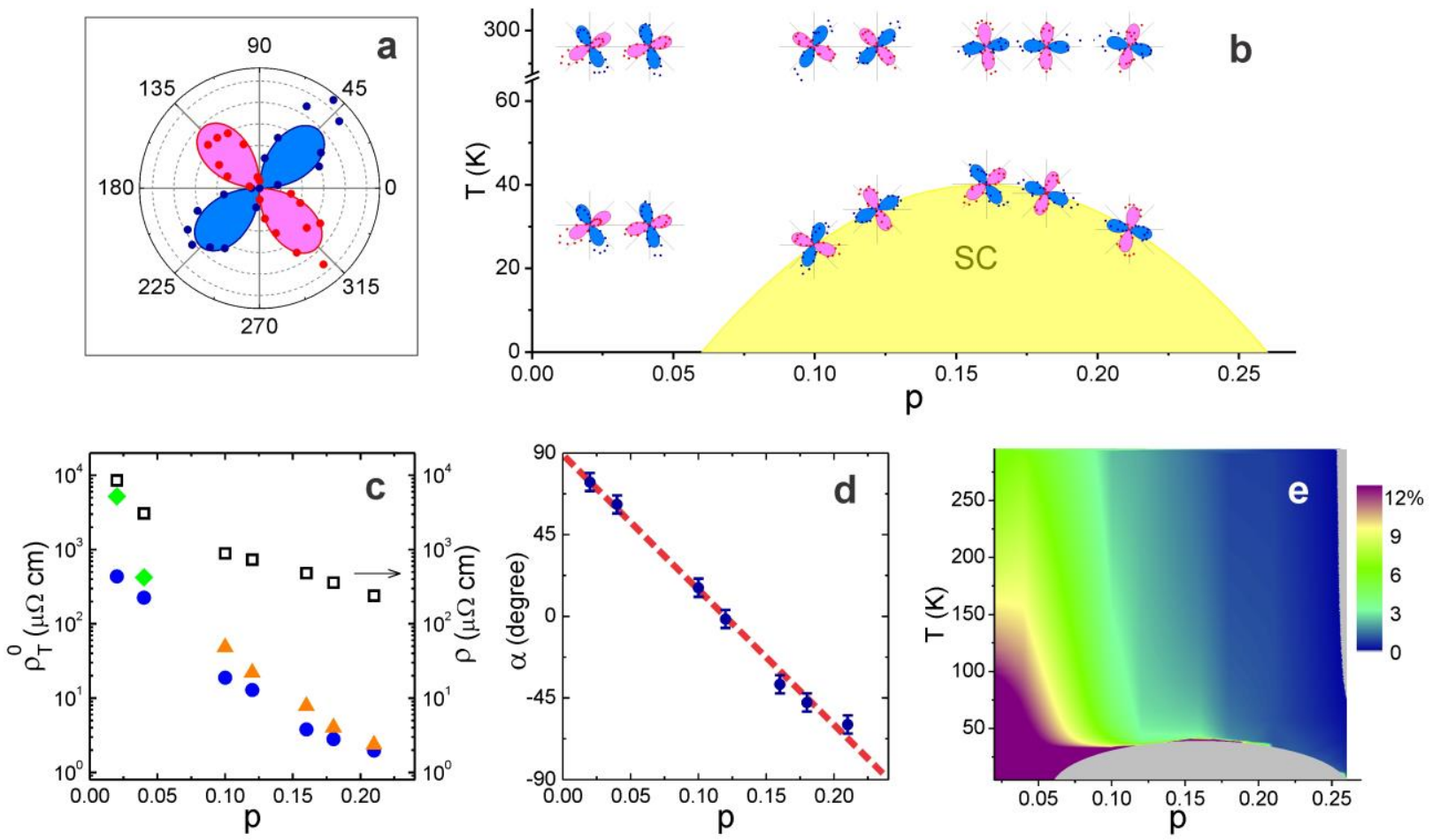

Figure 4 Angular, temperature and doping dependence of $\rho_{T}(\phi, T, p)$. a, An example of $\rho_{T}(\phi)$ dependence in polar coordinates (for LSCO film with $p=0.12$ at $T=295 \mathrm{~K}$ ). It resembles the 'cloverleaf' shape characteristic of $d$-orbital symmetry. The radial distance measures the magnitude of $\rho_{T}$, with the positive values in blue and negative in red. b, The phase diagram showing that the cloverleaf-shaped $\rho_{T}(\phi)$ dependence is universal for the entire phase space investigated here. (For better visibility, the radial extents are all normalized to the same size.) c, The doping dependence of $\bar{\rho}$ (right scale) and $\rho_{T} \mathrm{O}$ (left scale): open black squares, longitudinal resistivity $\bar{\rho}(p)$ measured at $T=295 \mathrm{~K}$; solid blue circles, $\rho_{T} \varrho(p)$ at $T=295 \mathrm{~K}$; solid yellow triangles, $\rho_{T}{ }^{\rho}(p)$ at $T=T_{c}$ (midpoint); solid green diamonds, $\rho_{T}{ }^{0}$ at $T=30 \mathrm{~K}$ is shown (in lieu of $T=T_{c}$ ) for the samples with $p=0.02$ and $p=0.04$ that are non-superconducting. $\mathbf{d}$, The doping dependence of the offset angle $\alpha$ measured at $T=295 \mathrm{~K}$. The angular resolution, and the upper limit on the error bar of $\alpha$, is $\pm 5^{\circ}$. e, The doping and temperature dependence of the 'nematicity' magnitude, $N \equiv \rho_{\mathrm{T}}^{0} / \bar{\rho}=\left|\left(\rho_{\mathrm{a}}-\rho_{\mathrm{b}}\right)\right| /\left(\rho_{\mathrm{a}}+\rho_{\mathrm{b}}\right)$. In the gray areas, the signal is below our noise floor. 


\section{METHODS}

\section{A. Molecular beam epitaxy synthesis}

Single-crystal LSCO thin films were synthesized on LSAO substrates by atomic-layer-by-layer molecular beam epitaxy (ALL-MBE) ${ }^{31-37}$ Films were grown by depositing $\mathrm{La}, \mathrm{Sr}$ and $\mathrm{Cu}$, shuttered sequentially to form full LSCO layers one at a time. The ozone partial pressure was kept at $p\left(\mathrm{O}_{3}\right)=10^{-6}$ Torr, and the substrate temperature was $T_{s}=700^{\circ} \mathrm{C}$. The growth process was monitored in situ by reflection high-energy electron diffraction (RHEED). The difractograms showed epitaxial growth, with the $\mathrm{Cu}-\mathrm{O}-\mathrm{Cu}$ bond direction in LSCO parallel to the Al-O-Al bond direction in LSAO, see Extended Data Fig. 1. The film thickness was controlled to be precisely 20 unit cells $(26 \mathrm{~nm})$ for all the samples shown here, although we have studied both thinner and thicker films as well for comparison. After the growth, underdoped LSCO films were annealed in vacuum at $T_{s}=300^{\circ} \mathrm{C}$ for several hours to eliminate excess interstitial oxygen ions. Overdoped films were annealed under $p\left(\mathrm{O}_{3}\right)=10^{-5}$ Torr at $T_{s}=700^{\circ} \mathrm{C}$ to minimize the concentration of oxygen vacancies. (This is the primary source of the difference ${ }^{4}$ between the nominal $\mathrm{Sr}$ doping level $x$ and the estimated mobile hole density $p$.) We have established this recipe for preserving the correct stoichiometry based on synthesis and study of over two thousand LSCO films in the last decade.

To illustrate the film quality and homogeneity, in Extended Data Fig. 2 we show the Meissner effect measurement in an optimally doped LSCO film using the mutual inductance technique. ${ }^{4,36}$ The in-phase signal measures the reactive response and shows the Meissner effect with a sharp onset at the temperature at which the resistivity drops to zero, $T_{c}=40.8 \mathrm{~K}$. The half-width-athalf-maximum of the peak in the out-of-phase (dissipative) response puts an upper limit on the variations of $T_{c}$ in this film, $\Delta T_{c}<0.1 \mathrm{~K}$, over a large area of $10 \times 10 \mathrm{~mm}^{2}$. This by itself rules any attribution of the observed phenomena, including in particular the peak in $V_{\mathrm{T}}$ near $T_{c}$, which is typically 1-2 orders of magnitude broader, to film inhomogeneity.

\section{B. Atomic steps on substrates studied by atomic force microscopy (AFM)}

The surfaces of LSAO substrates were polished perpendicular to the crystallographic [001] direction. However, due to inevitable small misalignment (a miscut angle $<0.5^{0}$ ), atomic steps occur at the surface of every substrate. Since the miscut orientation varies randomly from substrate to 
substrate, the alignments of atomic steps vary accordingly. We used an atomic force microscope to measure the orientation and density of atomic steps in every LSAO substrate prior to film growth and in LSCO films after the growth. The atomic steps in LSAO substrate carry over into the LSCO film, indicating layer-by-layer growth.

In principle, such atomic steps (and the induced anti-phase domain boundaries and dislocations in the film) break the rotation and mirror-plane symmetries of the crystal structure. Hence, we have investigated in detail whether there is a correlation between the step orientation and the direction in which the maximum of $\rho_{T}(\phi)$ occurs, but have found none (Extended Data Fig. 3), ruling out this explanation. The same argument rules out the decisive role of the strain anisotropy that arises when LSCO, that would be orthorhombic in the bulk, is constrained to be tetragonal by epitaxy.

\section{Lithography, contacts, misalignment}

We used a well-tested lithographic process to pattern our films into devices for transport measurements. The LSAO substrates are cut and polished with the edges parallel to the crystallographic [100], [010] and [001] directions with the accuracy better than $\pm 0.5^{0}$. We align the mask to the substrate edges under the microscope, and the resulting deviation of the lithographic $\theta=0^{0}$ direction from the crystallographic [001] orientation is less than $\pm 1^{0}$.

The films were etched by ion bombardment. To provide for good electric contacts, we deposited a $500 \mathrm{~nm}$ thick layer of $\mathrm{Au}$ on each contact pad. In the Extended Data Figs. 4a and 4b, we show examples of the dependences of $V_{T}$ and of the longitudinal voltage $\left(V_{x x}=V\right)$, respectively, on the probe current, measured in one sample at several temperatures. Both the $V-I$ and $V_{T^{-}} I$ characteristics are essentially linear up to $I=10 \mu \mathrm{A}$, at all temperatures above $T_{c}$. In our $\rho(T)$ and $\rho_{T}(T)$ measurements, we kept the excitation current at $I=2 \mu \mathrm{A}$, and the $V$ - $I$ relations were linear in every device. This rules out the possibility that $V_{T}$ could be a voltage offset due to contact imperfections.

Some misalignment between the pair of Hall contacts is practically inevitable in any transport experiment, but we have ruled this out as the origin of $V_{T}$ we observe, as follows. First, an upper limit on such a misalignment in our experiments is set by the precision in fabricating the lithographic mask, which is better than $\pm 1 \mu \mathrm{m}$. Since the distance between the longitudinal voltage 
contacts is $L=300 \mu \mathrm{m}$, the upper limit on $\rho_{T}$ caused by the misalignment is $\pm 0.3 \%$ of the value of $\rho$. However, the ratio $\rho_{T} / \rho$ we observed is much larger than this; e.g., for $p=0.1, \rho_{T} / \rho=2.4 \%$ at $T=295 \mathrm{~K}$ while it exceeds $20 \%$ near $T_{c}$. Second, in this scenario one would expect $\rho_{T}=A \rho$ with a fixed coefficient $A(<0.003)$; in contrast, the $\rho_{T}(T, \phi, p)$ and $\rho(T, \phi, p)$ dependences that we observe are qualitatively different from one another. This should be quite obvious from e.g., Extended Data Fig. 5, showing that the $\rho_{T} / \rho$ ratio varies with both $T$ and $p$, as well as from Fig. 3, showing that $\rho_{T} / \rho$ also has a strong and very specific $\phi$ dependence. Third, these misalignments and the ratio $\rho_{T} / \rho$ could be random for different devices within one lithographic mask but are repeatable in different films, since all were patterned using the same mask. Hence, within this scenario one would not expect any systematic dependence of $\rho_{T}$ on $\phi$, and none on the doping level $p$ - contrary to our observations (see e.g., Extended Data Fig. 6). Fourth, this certainly cannot explain the observed angular dependence of the longitudinal resistivity, $\rho=\bar{\rho}+\rho_{T}{ }^{0}$ $\cos [2(\phi-\alpha)]$, as shown in Fig. 3.

\section{Angular dependence of $\rho$ and $\rho_{T}$}

To study the angular dependence of $\rho$ and $\rho_{T}$, we designed a "sun-beam" lithography pattern shown in Fig. 2a. The angle $\phi$ between the current-carrying strip and the crystal [100] direction is increased from $0^{0}$ to $360^{\circ}$ in the steps of $\Delta \phi=10^{\circ}$. The width of each strip is $100 \mu \mathrm{m}$. Six voltage contact pads are attached to each strip, so that $\rho_{T}$ can be measured at three locations spaced 300 $\mu \mathrm{m}$ apart, and averaged to reduce random variations due to lithography, substrate polishing defects, etc. By switching the connections to the contact pads in an external electronic box, we can measure $\rho$ of the same device. Hence, we can map out the complete angular dependence of $\rho$ and $\rho_{T}$ with the accuracy of $\pm 5^{0}$ on a single sample. This eliminates sample-to-sample variations due to inevitable differences in the substrate surface miscuts, the deposition parameters, film nucleation and growth, etc.

To rule out any artifacts due to lithography, we have also devised and performed another type of experiment in which there are no lithographic steps whatsoever. We had a shadow mask micromachined to delineate a ring of 36 contact pads. Then we just evaporate gold contacts, at room temperature, through the shadow mask, as illustrated in Extended Data Fig. 7a. We then make the angle-resolved transverse resistance (ARTR) measurements using two pairs of contacts in a 
cross. As shown in Extended Data Fig. 7b, the results came out essentially the same, ruling out any role of lithography. Moreover, we have reproduced the same experiment both on our thin films and on bulk LSCO single crystals from a different source (J. Wu, A. T. Bollinger, G. Gu and I. Bozovic, unpublished), ruling out any artifacts due to substrates, films, epitaxy, lithography, etc. While this approach is technically simpler than film patterning, the data are affected by the geometry of the film, as shown in Extended Data Fig. 7c and 7d. The advantage of our scheme using the 'sunbeam' pattern is that the current direction is uniform and guided by the strip, and hence the analysis of the data is much simpler - one needs no modeling whatsoever.

\section{E. Low-temperature transport measurements}

A difference in temperature at the two transverse contacts could also generate a transverse voltage, but we have ruled this out. Such an effect would be very small, it would be even with respect to the current reversal while $V_{T}$ that we measure is odd, the angular dependence would not be $d$-wave like, and there would be no systematic doping dependence. Moreover, the temperature gradient across our $1 \mathrm{~cm} \times 1 \mathrm{~cm}$ sample is less than $1 \mathrm{mK}$.

To avoid device self-heating, we keep the excitation current density for transport measurements low enough, at $80 \mathrm{~A} / \mathrm{cm}^{2}$. This corresponds to the probe current $I_{x}=2 \mu \mathrm{A}$, while (as shown in Extended Data Fig. $4 \mathrm{a}$ and $4 \mathrm{~b}$ ) for both $V$ and $V_{T}$ the $I-V$ relations are essentially linear up to at least $10 \mu \mathrm{A}$.

We used the Helium-4 and Helium-3 cryogenics to reach temperatures down to $T=4.2 \mathrm{~K}$ and $T$ $=0.3 \mathrm{~K}$, respectively. In both cases, the sample is surrounded by exchange gas and the temperature stability is better than $\pm 1 \mathrm{mK}$.

\section{F. Symmetry}

The crystal structure of our LSCO films is approximately tetragonal at all doping levels; orthorhombic distortions are suppressed because the films are thin and epitaxially anchored to the tetragonal LSAO substrates (see Extended Data Fig. 1). To be quantitative, X-ray diffraction data in twice thicker LSCO films grown on LSAO substrates by ALL-MBE show the in-plane orthorhombic distortion of just $0.08 \%$ in insulating, $0.04 \%$ in optimally doped, and $0.01 \%$ in overdo-

ped metallic LSCO films, respectively. ${ }^{33}$ Hence, even at the lowest doping levels, the distortion in films is smaller by at least a factor of 20 than in the corresponding bulk samples. (Note that if 
$\mathrm{Q}$ is the distortion amplitude, in the simple Peierls model the total CDW condensation energy scales as $Q^{2} \ln Q$, so it would be reduced by three orders of magnitude.)

On the other hand, the symmetry with respect to the horizontal mirror plane $\hat{\sigma}_{\mathrm{h}}=\hat{\sigma}_{\mathrm{z}}$, which maps $z$ to $-z$, is broken externally by epitaxial strain and/or by the long-range Madelung interaction with the substrate. Breaking of the discrete translational symmetry, if present, would not show up in our macroscopic measurements.

Hence, for our discussion here, the relevant spatial symmetry group is the point group $\mathbf{C}_{4 \mathrm{v}}$ (in the Schoenfliss notation) or $4 \mathrm{~mm}$ (in the International Hermann-Mauguin notation.) $\mathbf{C}_{4 \mathrm{v}}=\mathbf{C}_{4}+\hat{\sigma}_{\mathrm{v}} \mathbf{C}_{4}$, where $\mathbf{C}_{4}=\left\{\mathrm{E}, \hat{\mathrm{C}}_{4}, \hat{\mathrm{C}}_{2}, \hat{\mathrm{C}}_{4}{ }^{-1}\right\}, \hat{\mathrm{C}}_{4}$ and $\hat{\mathrm{C}}_{2}$ are rotations by $\pi / 2$ and $\pi$, respectively, around the $\mathrm{z}-$ axis, and $\hat{\sigma}_{\mathrm{v}}=\hat{\sigma}_{\mathrm{y}}$ is the 'vertical' mirror reflection in the $x z$ plane. The $\phi$-dependence of $\rho_{T}$ (Figs. 2, 3 and 4) shows that $\mathbf{C}_{4}$-symmetry is broken. If the offset angle $\alpha=0$, the $\hat{\sigma}_{\mathrm{v}}$ mirror symmetry is preserved. This corresponds to the $\mathrm{B}_{2}$ irreducible representation of $\mathbf{C}_{4 \mathrm{v}}$, commonly referred to as the ' $d$-wave' symmetry.

An important open question is whether we have (and are averaging over) domains of different orientations. We hope to resolve this in the future by studying much smaller devices, down to the submicron scale.

\section{G. Transverse voltage due to anisotropic resistivity}

In general, $\boldsymbol{E}=\rho \boldsymbol{J}$, where $\rho$ is a tensor. Restricting ourselves to in-plane properties, for an orthorhombic material

$$
\rho=\left(\begin{array}{cc}
\rho_{a} & 0 \\
0 & \rho_{b}
\end{array}\right)
$$

in the coordinate system $\mathbf{e}_{\mathbf{a}}, \mathbf{e}_{\mathbf{b}}$ defined by the principal axes, which for simplicity in this section we assume to coincide with the crystallographic [ $\left[\begin{array}{lll}1 & 0 & 0\end{array}\right]$ and $\left[\begin{array}{lll}0 & 1 & 0\end{array}\right]$ directions, respectively. Let us define $\bar{\rho}=\left(\rho_{\mathrm{a}}+\rho_{\mathrm{b}}\right) / 2$ and $\Delta \rho=\left(\rho_{\mathrm{a}}-\rho_{\mathrm{b}}\right) / 2$; then $\rho_{\mathrm{a}}=\bar{\rho}+\Delta \rho$ and $\rho_{\mathrm{b}}=\bar{\rho}-\Delta \rho$. Now, if we ro-

tate the axes by an angle $\phi$, so that $\mathbf{e}_{\mathbf{x}}=\hat{\mathrm{C}}_{\phi} \mathbf{e}_{\mathbf{a}}$ and $\mathbf{e}_{\mathbf{y}}=\hat{\mathrm{C}}_{\phi} \mathbf{e}_{\mathbf{b}}$, in the coordinate system $\mathbf{e}_{\mathbf{x}}, \mathbf{e}_{\mathbf{y}}$, the matrix $\rho$ changes and becomes:

$\hat{\mathrm{C}}_{\phi} \rho \hat{\mathrm{C}}_{\phi}{ }^{-1}=\left(\begin{array}{cc}\cos \varphi & -\sin \varphi \\ \sin \varphi & \cos \varphi\end{array}\right)\left(\begin{array}{cc}\rho+\Delta \rho & 0 \\ 0 & \rho-\Delta \rho\end{array}\right)\left(\begin{array}{cc}\cos \varphi & \sin \varphi \\ -\sin \varphi & \cos \varphi\end{array}\right)=\left(\begin{array}{cc}\rho+\Delta \rho \cos (2 \varphi) & \Delta \rho \sin (2 \varphi) \\ \Delta \rho \sin (2 \varphi) & \rho-\Delta \rho \cos (2 \varphi)\end{array}\right)$ 
while $\mathbf{E}=\left(\begin{array}{c}E_{x} \\ E_{y}\end{array}\right)$ and $\mathbf{J}=\left(\begin{array}{c}J_{x} \\ J_{y}\end{array}\right)$

If we now force the current to flow along $\mathbf{e}_{\mathbf{x}}$, i.e., at angle $\phi$ with respect to $\mathbf{e}_{\mathbf{a}}$, we get $\mathbf{J}=\left(\begin{array}{l}J \\ 0\end{array}\right)$ and hence

$$
\begin{aligned}
& E_{x} / J=\rho_{x x}=\bar{\rho}+\Delta \rho \cos (2 \phi) \\
& E_{y} / J=\rho_{\mathrm{xy}}=\Delta \rho \sin (2 \phi)
\end{aligned}
$$

Therefore, in every orthorhombic material, as long as the current is not aligned with one of the principal axes there must occur a spontaneous transverse voltage, and it will show the $d$-wavelike, $\sin (2 \phi)$ symmetry. Note that this occurs in zero magnetic field, and has nothing to do with normal or anomalous Hall effect, ferromagnetism, skew spin scattering and lateral hops, Berry phase, loop currents, orbital antiferromagnetism, Rashba spin-orbit coupling, etc. On the other hand, this bears relation to the previous inferences ${ }^{38-47}$ about anisotropy of the mean-free path and the scattering rate in cuprates based on angle-resolved magnetoresistance measurements on $\mathrm{Tl}_{2} \mathrm{Ba}_{2} \mathrm{CuO}_{6+\mathrm{d}}$ and LSCO. The important novel observations here are that anisotropy is nematic, detectable at every doping and from $T_{c}$ up to the room temperature, apparently not phase-locked (on macroscopic scale of the present experiment) to the crystal axes, and growing dramatically in the fluctuation region near $T_{c}$.

\section{H. Nematic order parameter}

In view of the above, we propose that the 'nematic metal' state can be characterized by the order parameter with the (relative) magnitude

$$
N \equiv \rho_{\mathrm{T}}^{0} / \bar{\rho}=\left|\left(\rho_{\mathrm{a}}-\rho_{\mathrm{b}}\right)\right| /\left(\rho_{\mathrm{a}}+\rho_{\mathrm{b}}\right) .
$$

While the two expressions are equivalent mathematically, the first one has a great advantage when it comes to the actual measurements. If the difference between $\rho_{\mathrm{a}}$ and $\rho_{\mathrm{b}}$ is small compared to the large 'background', it may be obscured by the device-to-device uncertainty due to random geometrical and other variations. In contrast, $\rho_{\mathrm{T}}{ }^{0}$ is free of such a background, and can be measured more accurately. This can be seen very clearly by comparing the top and bottom panels in Figs. 2b-2d. (The analogous large increase of sensitivity achieved by measuring the 
transverse rather than the longitudinal resistance has been demonstrated and exploited in Ref. 37).

In the case of a purely electronic ' $x y$ nematicity', the effect is also characterized by a 'director' $\boldsymbol{n}$, of unit length, which defines an axis orientation (but has no sense of direction). This axis can be chosen e.g., at the angle $\alpha$, i.e., along the direction of the maximal resistivity. In general, in our LSCO films we observe that $\boldsymbol{n}$ is not aligned with the crystal axes (or diagonals). Note that due to $\mathbf{C}_{4 \mathbf{v}}$ symmetry, there are four equivalent choices of $\alpha$ in a given sample (two spaced at $90^{\circ}$ and two more from the mirror symmetry). In Fig. 4d, we have chosen these along the essentially straight $\alpha(p)$ line, for $T=295 \mathrm{~K}$; however, once this choice is made, it presumably stays fixed at lower temperatures. Then, within a certain doping range, we observe that $\boldsymbol{n}$ rotates near $T_{c}$, in the region of superconducting fluctuations, see Fig. 4 and Extended Data Fig. 8. We also show that $\alpha$ does not depend on the direction of the substrate/film steps and terraces (Extended Data Fig. 3) and is not affected by moderate magnetic fields (Extended Data Fig. 9). However, it gets pinned down to one of the principal crystal axes when a significant $(\sim 1 \%)$ orthorhombic distortion is induced by epitaxy (Extended Data Fig. 10).

Alternatively, instead of $N$ one could utilize the 'nematic angle' $\Theta \equiv \tan ^{-1} N$. It also has a clear physical meaning: in analogy with the familiar Hall angle, $\Theta$ is the angle between the directions of the (total, resulting) electric field $\boldsymbol{E}$ and the current $\boldsymbol{J}$. Like $N$, this 'nematic angle' $\Theta$ characterizes the relative magnitude, or the 'degree' of nematicity, and it should not be confused with the director angle $\alpha$.

\section{Statistics, reproducibility, and (in)homogeneity}

Two lithographic patterns were used. The first ${ }^{35,37}$ contains a single strip, $10 \mathrm{~mm}$ long, provided with 32 pairs of symmetric contacts, see Extended Data Fig. 6. In this case, the strip is always oriented parallel to the crystallographic [100] direction, to within $\pm 1 \%$ accuracy. We have measured $\rho_{T}$ in over 3,000 such devices at discrete temperatures (e.g., $T=40 \mathrm{~K}, 50 \mathrm{~K}, \ldots, 300 \mathrm{~K}$ ). In about 200 devices, we measured $\rho_{T}(T)$ continuously from $T=300 \mathrm{~K}$ to $T=4 \mathrm{~K}$.

The second pattern is the sunbeam structure introduced in this work and shown in Fig. 2a, which we have used to study the angular dependence of $\rho_{T}$ and $\rho$. In such sunbeam-patterned LSCO 
films, we measured $\rho_{T}$ at $T=295 \mathrm{~K}$ in approximately 2,000 devices, while the continuous $\rho_{T}(T)$ dependence was measured in about 1,000 devices.

As pointed out in Ref. 48, an asymmetric inhomogeneity in a superconducting sample can produce nonzero $V_{T}$, peaked at $T_{c}$ (midpoint). That is a plausible explanation for polycrystalline samples $^{48-51}$, in the flux-flow regime. It is not very plausible for our single-crystal films, which are homogeneous on the $0.1 \mathrm{~K}$ scale over the $10 \times 10 \mathrm{~mm}^{2}$ area (Extended Data Fig. 1), even if just a single device is considered. It becomes quite improbable when all of 31 devices in a linear library show essentially the same $N$ and $\alpha$, (see Extended Data Fig. 6) or when all 108 devices in a sunbeam pattern follow the $\sin (2 \phi)$ angular dependence - at every doping, in every film, at every temperature. More is different here; our large statistics allows for definitive statements. The systematic angle, doping, and temperature dependences reported here rule out random extrinsic factors and point to behavior intrinsic to LSCO films.

More detailed information is provided in the extensive source data files available online.

\section{References}

31. Božović, I. Atomic-layer engineering of superconducting oxides: Yesterday, today, tomorrow. IEEE Trans. Appl. Supercond. 11, 2686-2695 (2001).

32. Gozar, A. et al. Interface superconductivity between a metal and a Mott insulator. Nature 455, 782785 (2008).

33. Butko, V., Logvenov, G., Božović, N., Radović, Z. \& Božović, I. Madelung strain in cuprate superconductors - a route to enhancement of the critical temperature. Adv. Mater. 21, 3644-3688 (2009).

34. Bollinger, A. T. et al. Superconductor-insulator transition in $\mathrm{La}_{2-x} \mathrm{Sr}_{x} \mathrm{CuO}_{4}$ at the pair quantum resistance. Nature 472, 458-460 (2011).

35. Wu, J. et al. Anomalous independence of interface superconductivity on carrier density. Nature Mater. 12, 877-881 (2013).

36. He, X., Gozar, A., Sundling, R. and Božović, I. High-precision measurement of magnetic penetration depth in superconducting films. Rev. Sci. Instr. 87, 113903 (2016).

37. Wu, J., Bollinger, A. T., Sun Y.-J. \& Božović, I. Hall Effect in quantum critical charge-cluster glass. Proc. Natl. Acad. Sci. USA 113, 4284 (2016).

38. Hussey, N. E. et al. Angular dependence of the c-axis normal state magnetoresistance in single crystal $\mathrm{Tl}_{2} \mathrm{Ba}_{2} \mathrm{CuO}_{6}$. Phys. Rev. Lett. 76, 122-125 (1996).

39. Hussey, N. E. Low-energy quasi-particles in high- $T_{c}$ cuprates. Adv. Phys. 51, 1685-1771 (2002).

40. Hussey, N. E. The normal state scattering rate in high- $T_{c}$ cuprates. Eur. Phys. J. B 31, 495-507 (2003). 
41. Nakamae, S. et al. Electronic ground state of heavily overdoped nonsuperconducting $\mathrm{La}_{2-x} \mathrm{Sr}_{x} \mathrm{CuO}_{4}$. Phys. Rev. B 68, 100502(R) (2003).

42. Abdel-Jawad, M. et al. Anisotropic scattering and anomalous normal-state transport in a hightemperature superconductor. Nature Phys. 2, 821-825 (2006).

43. Abdel-Jawad, M. et al. Correlation between the superconducting transition temperature and anisotropic quasiparticle scattering in $\mathrm{Tl}_{2} \mathrm{Ba}_{2} \mathrm{CuO}_{6+d}$. Phys. Rev. Lett. 99, 107002 (2007).

44. Analytis, J. G., Abdel-Jawad, M., Balicas, L., French, M. M. J. \& Hussey, N. E. Angle-dependent magnetoresistance measurements in $\mathrm{Tl}_{2} \mathrm{Ba}_{2} \mathrm{CuO}_{6+d}$ and the need for anisotropic scattering. Phys. Rev. B 76, 104523 (2007).

45. Narduzzo, A. et al. Violation of the isotropic mean free path approximation for overdoped $L_{2}$ ${ }_{x} \mathrm{Sr}_{x} \mathrm{CuO}_{4}$. Phys. Rev. B 77, 220502(R) (2008).

46. Kokalj, J. \& McKenzie, R. H. Consistent description of the metallic phase of overdoped cuprate superconductors as an anisotropic marginal Fermi liquid. Phys. Rev. Lett. 107, 147001 (2011).

47. Kokalj, J., Hussey, N. E. \& McKenzie, R. H. Transport properties of the metallic state of overdoped cuprate superconductors from an anisotropic marginal Fermi liquid model. Phys. Rev. B 86, 045132 (2012).

48. Segal, A., Karpovski, M. \& Gerber, A. Inhomogeneity and transverse voltage in superconductors. Phys. Rev. B 83, 094531 (2011).

49. Francavilla, T. L., Cukauskas, E. J., Allen, L. H. \& Broussard, P. R. Observation of a transverse voltage in the mixed state of YBCO thin films. IEEE Trans. Appl. Supercond. 5, 1717-1720 (1995).

50. Vašek, P., Janeček, I. \& Plecháček, V. Intrinsic pinning and guided motion of vortices in high- $T_{c}$ superconductors. Physica C 247, 381-384 (1995).

51. Da Luz, M. S. et al. Observation of asymmetric transverse voltage in granular high- $\mathrm{T}_{\mathrm{c}}$ superconductors. Physica C 419, 71-78 (2005).

\section{Data Availability Statement}

All the data used to produce the figures in the main text as well as in the Extended Data are available online as Source Data files in MS Excel format. 


\section{Extended Data figures}

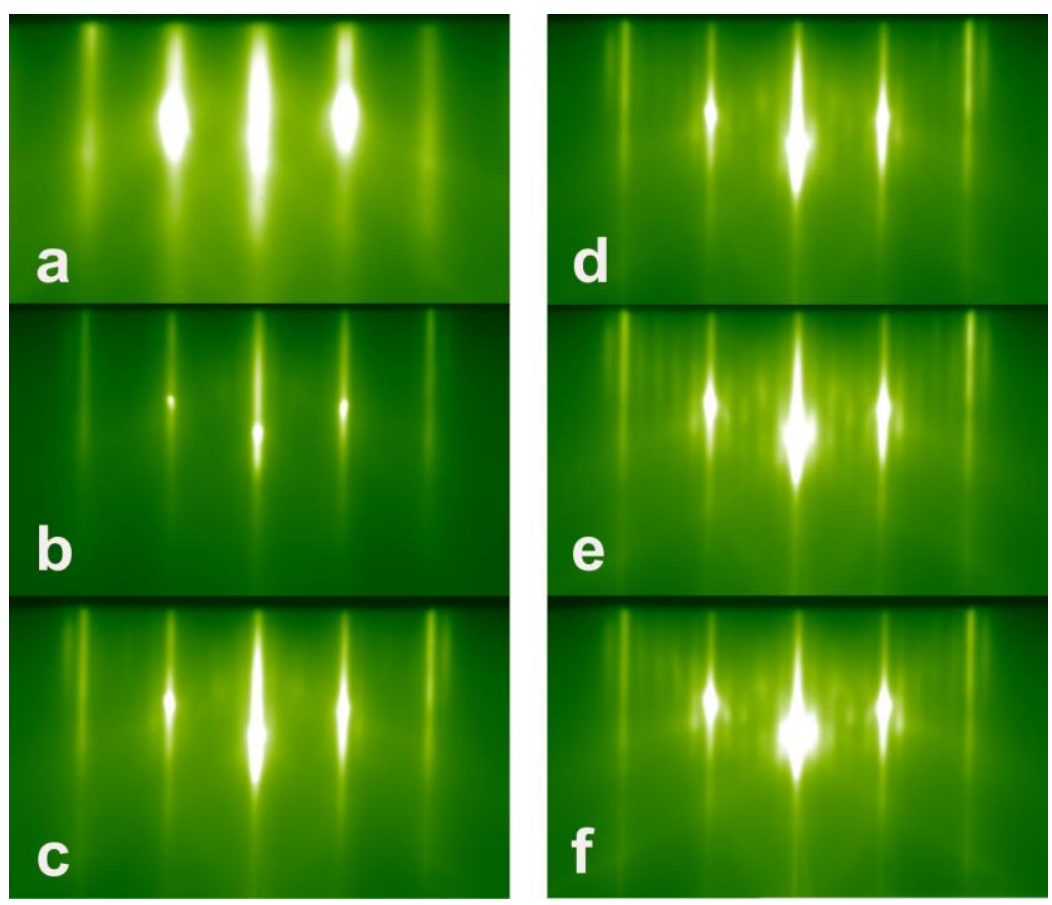

Extended Data Figure 1 RHEED images taken during growth of an optimally doped ( $p=0.16$ ) LSCO film. a, A diffraction pattern from LSAO substrate just before growth. $\mathbf{b}$, after the first LSCO layer $(0.66 \mathrm{~nm}$ thick = one-half the $c$-axis lattice constant). c, after the second LSCO layer. d, after the third LSCO layer. e, after the fifth LSCO layer. $\mathbf{f}$, after the twentieth LSCO layer.

Very strong specular reflection indicates that the surface is very flat even on the scale of the electron wavelength $(0.1 \AA)$. Long and narrow diffraction streaks indicate high film crystallinity (with some terracing that originates from a slight miscut of the substrate). The LSCO film is epitaxially constrained by the tetragonal LSAO substrate; the in-plane lattice constants are the same, and the $\mathrm{Cu}-\mathrm{O}-\mathrm{Cu}$ direction in LSCO is parallel to the AlO-Al direction in LSAO, i.e., the crystallographic [100] direction. 

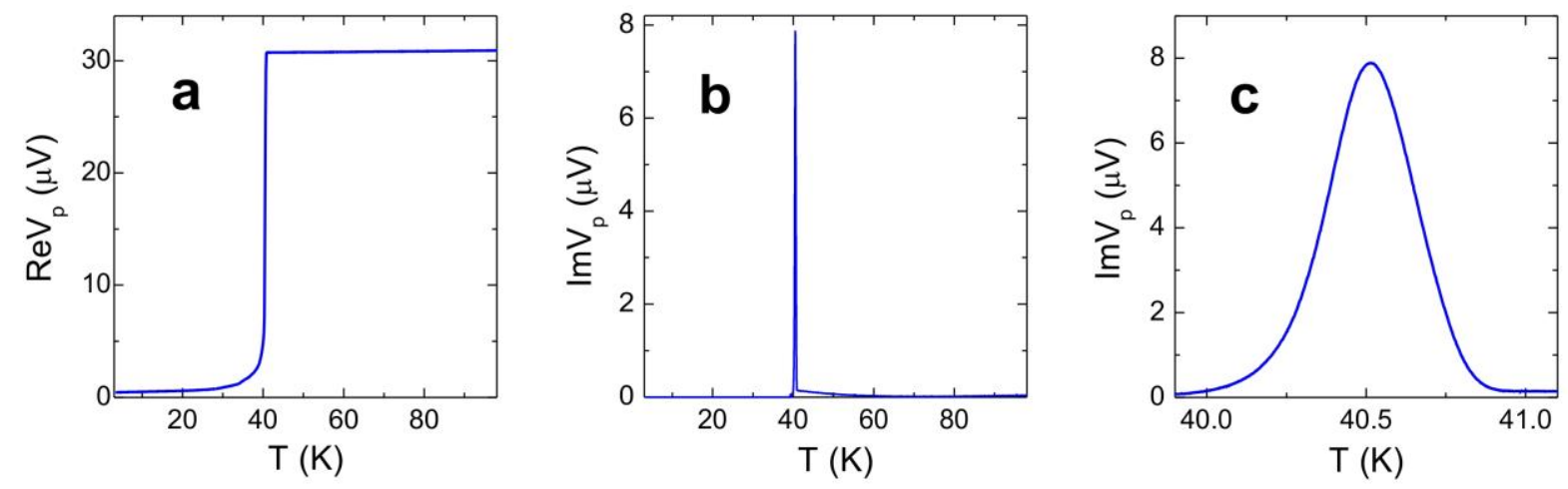

Extended Data Figure 2 $\mid$ Inductance data for an optimally doped $(p=0.16)$ LSCO film show remarkable sample homogeneity. a, The in-phase component of $V_{p}$, the voltage across the pickup coil (proportional to the mutual inductance). It shows diamagnetic screening (the Meissner effect) when the film becomes superconducting. $\mathbf{b}$, The imaginary part of $V_{p}$ in the same film. c, The same, magnified near $T_{c}$. If there were two peaks separated by more than one half-width-at-half-maximum, these would be clearly resolved. Hence, in this film (of $10 \times 10 \mathrm{~mm}^{2}$ area) $T_{c}$ is uniform to within $\pm 0.1 \mathrm{~K}$. 

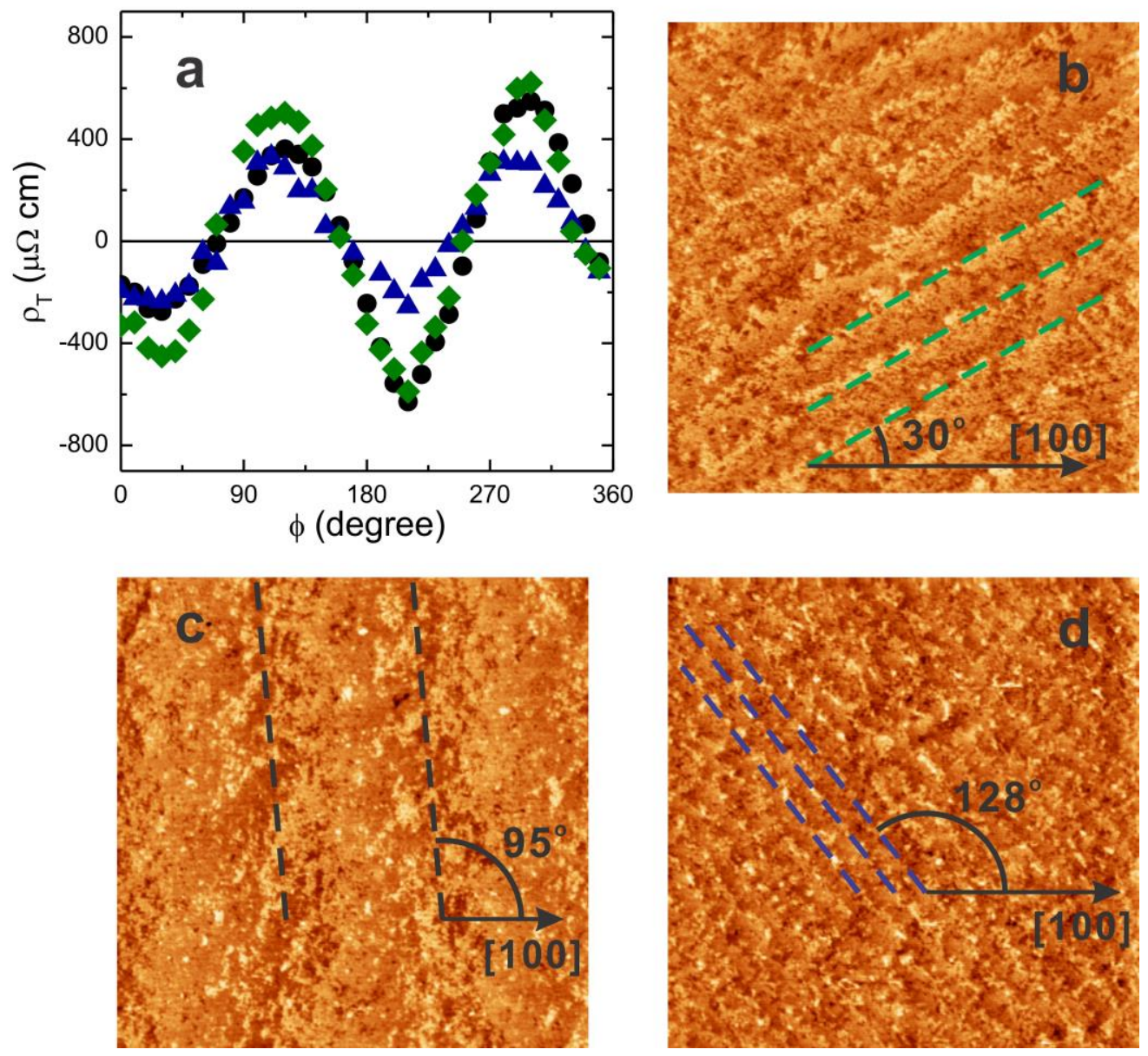

Extended Data Figure 3 The offset angle of $\rho_{T}(\phi)$ is not related to the substrate steps. a, The $\rho_{T}(\phi)$ data for three LSCO films of the same doping level $(p=0.02)$ and taken at the same temperature $(T=295 \mathrm{~K})$. b, $\mathbf{c}$, and $\mathbf{d}$, The atomic force microscope (AFM) images of the same three films show terraces and steps that originate from the substrate miscut and are preserved in the films. The scale is the same for all three images, $3 \mu \mathrm{m} \times 3 \mu \mathrm{m}$. The steps are highlighted using green, black and blue dashed lines, with the colors corresponding to those in panel a. The step density and orientation vary a lot from one film to another, while the nematicity magnitude and direction are similar. 

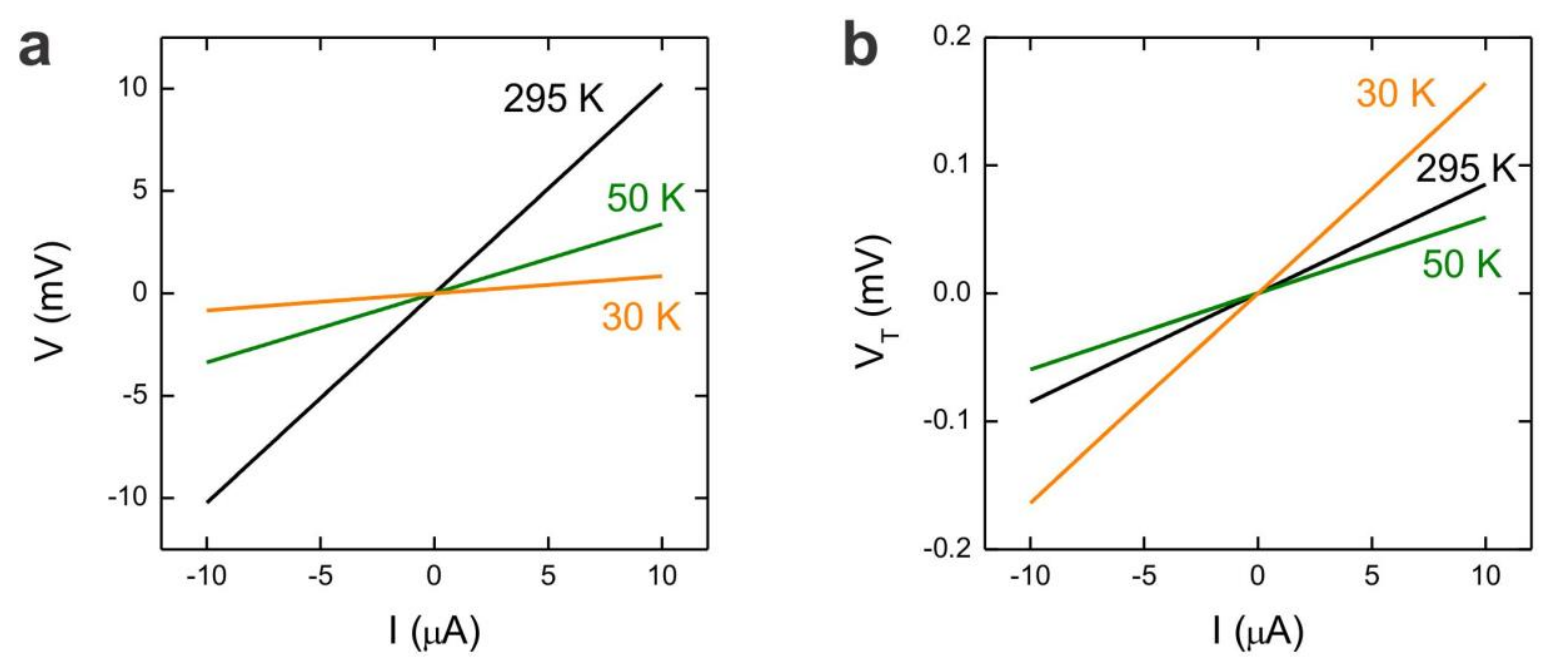

Extended Data Figure 4 | Examples of current-voltage characteristics in LSCO films grown by ALL-MBE. a, The dependence of the longitudinal voltage $V$ on the probe current $I$, at $T=30, T=50$ and $T=295 \mathrm{~K}$, respectively, in an LSCO film with $p=$ 0.10 . b. The dependence of the transverse voltage $V_{T}$ on the probe current $I$, measured at the same temperatures. Both $I-V$ and $I-V_{T}$ characteristics are strictly linear in the current range of interest. All other data shown in the paper are taken with a low probe current, $I=2 \mathrm{~mA}$ (which corresponds to the current density of $80 \mathrm{~A} / \mathrm{cm}^{2}$ ) and strictly ohmic contacts. 

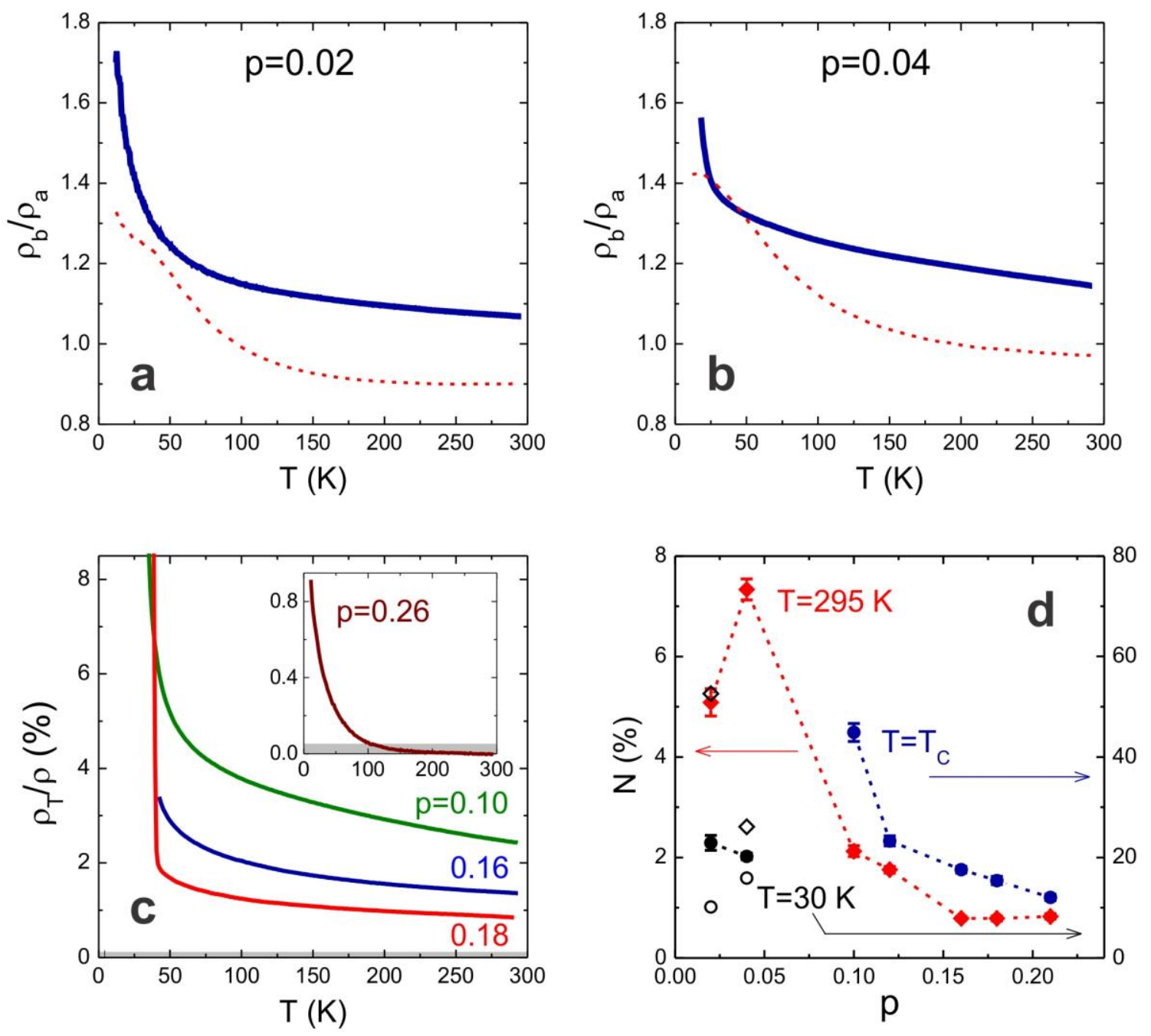

Extended Data Figure 5| 'Nematicity' in LSCO films and bulk crystals. a, The anisotropy in resistivity expressed as the ratio $\rho_{\mathrm{b}} / \rho_{\mathrm{a}}$. Blue solid curve: our data from a heavily underdoped ( $p=0.02)$ LSCO film. Red dashed line: data measured on bulk LSCO crystal with the same doping, reported in Ref. 8. b, The same as in panel a, except for $p=0.04$. c, The $\rho_{\mathrm{T}} / \rho$ ratio for several representative doping levels, underdoped $(p=$ $0.10)$, optimally doped $(p=0.16)$, and overdoped $(p=0.18)$. In all cases, there is a substantial, concave $T$ dependence with a fast rise in the region of superconducting fluctuations (not expected for misaligned contacts or, equivalently, for current path meandering due to sample inhomogeneity). The gray bar shows the noise floor. Inset: In heavily overdoped, non-superconducting ( $p=0.26)$ LSCO film, $\rho_{\text {T }}$ vanishes at $100-150 \mathrm{~K}$. d, Doping dependence of the 'nematicity' magnitude, $N \equiv \rho_{\mathrm{T}}{ }^{0} / \bar{\rho}=\left|\left(\rho_{\mathrm{a}}-\rho_{\mathrm{b}}\right)\right| /\left(\rho_{\mathrm{a}}+\rho_{\mathrm{b}}\right)$. Solid red diamonds, the data taken at $T=295 \mathrm{~K}$; solid blue circles, at $T=T_{c}$ (midpoint); solid black circles, at $T=30 \mathrm{~K}$. Open black diamonds, the data from Ref. 8, at $T=295 \mathrm{~K}$; open black circles, at $T=30 \mathrm{~K}$. The error bars represent the uncertainty in fitting $\rho_{T}(\phi)$. 

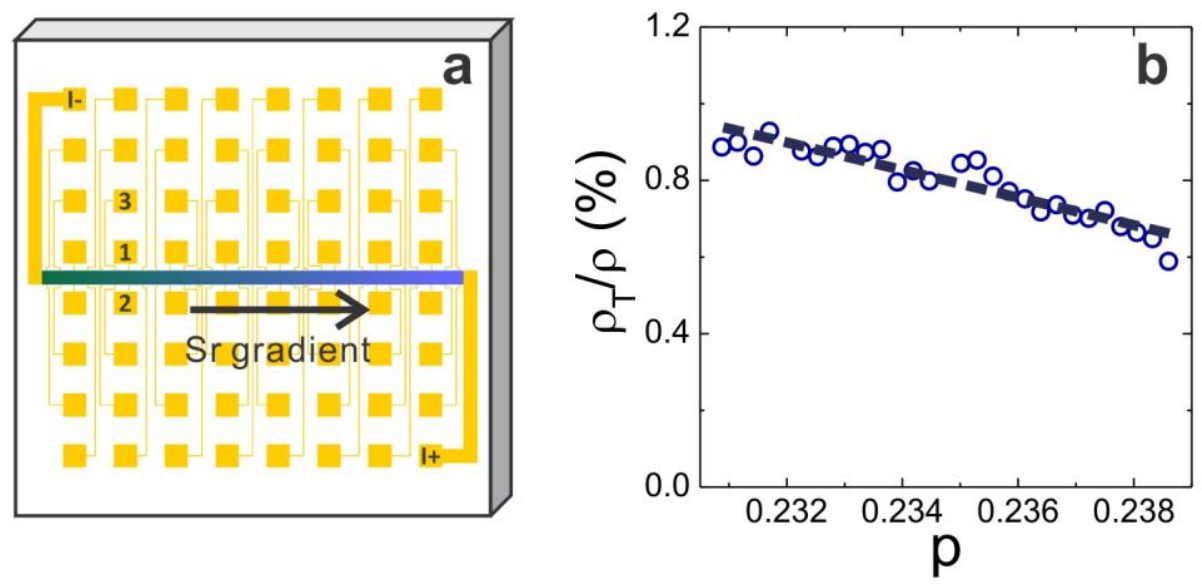

Extended Data Figure 6 | Systematic doping dependence of 'nematicity' in LSCO films rules out inhomogeneity as the source of transverse voltage. a, The lithography pattern we used ${ }^{35,37}$ to fabricate linear combinatorial libraries for measurements of longitudinal and transverse resistances. The central horizontal strip (300 $\mu \mathrm{m}$ wide and $10 \mathrm{~mm}$ long) is aligned with a gradient $(4 \%$ per $10 \mathrm{~mm})$ in Sr doping level, and is connected to 64 gold contact pads. This enables simultaneous measurements of $\rho$ in 30 'pixels' and $\rho_{T}$ in 31 'pixels', with extremely fine $(\Delta p=0.0003)$ steps in doping between two consecutive pixels. b. The 'nematicity' ratio shows a smooth and monotonous dependence on doping. Open blue circles: the measured data. Black dashed line: a linear fit to the data. 

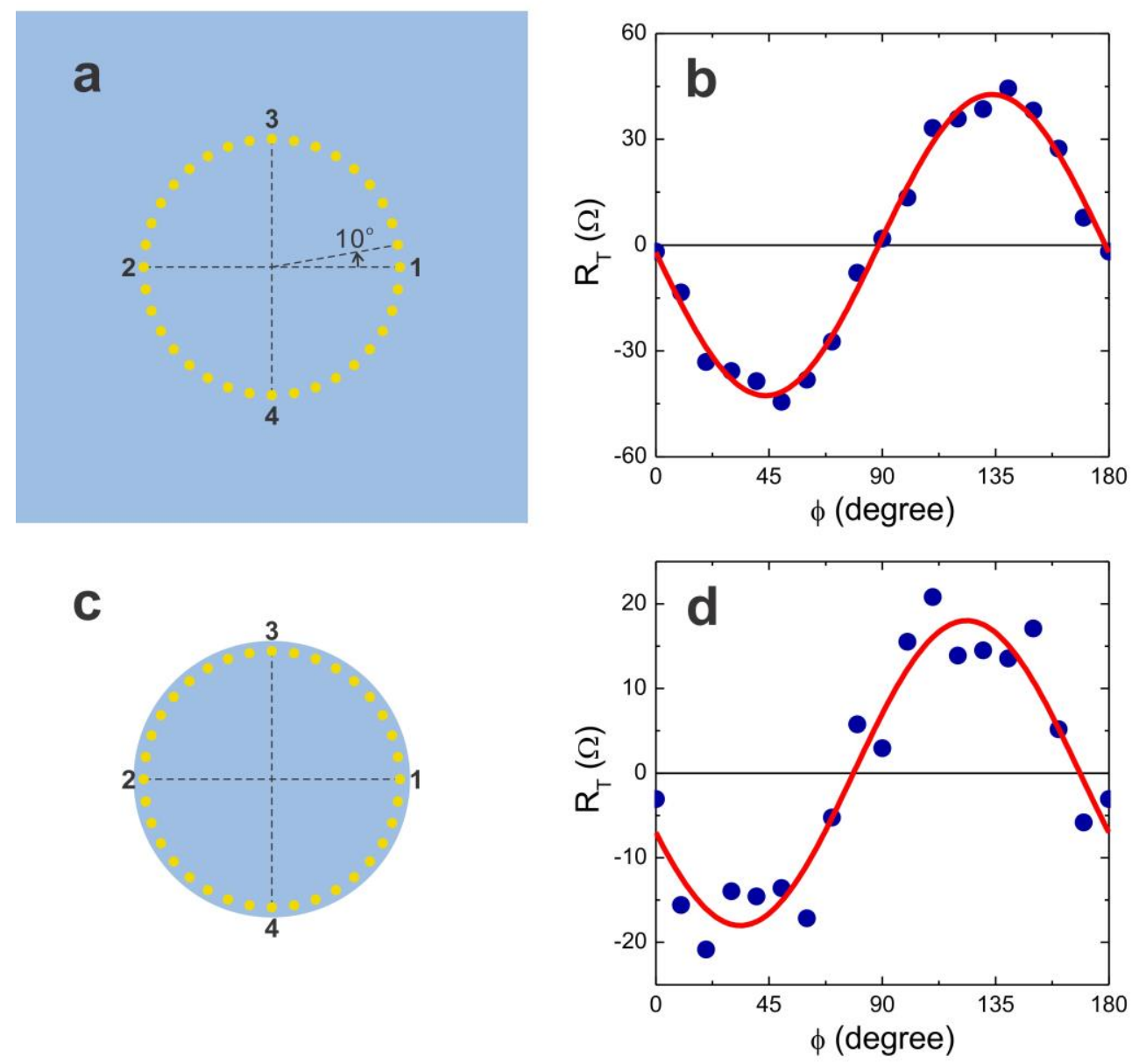

\section{Extended Data Figure 7 | Spontaneous transverse voltage measured using con-} tacts deposited through a shadow mask. a, A ring of 36 contact pads (yellow solid circles) deposited on an as-grown $p=0.04$ film (light blue square) by evaporating gold through a micro-machined shadow mask, without any lithographic steps. The transverse resistance $R_{T}$ is measured using a set of four contacts in a cross, e.g., by running the current through contacts 1 and 2 and measuring the voltage at contacts 3 and 4 , and analogously for other angles, in steps of $10^{\circ}$. The area of the LSCO film is $10 \times 10 \mathrm{~mm}^{2}$, the diameter of the circle is $5 \mathrm{~mm}$, and the diameter of the contact pads $0.2 \mathrm{~mm}$. $\mathbf{b}$, The resulting angular dependence of $R_{T}$ measured at $T=295 \mathrm{~K}$ in LSCO film doped to $p=$ 0.04 . c, The film outside of the central circle was etched away and $R_{T}(\phi)$ was measured again at $T=295 \mathrm{~K}$. The diameter of the remaining circle is $5.5 \mathrm{~mm}$. d, The resulting angular dependence of $R_{T}$.

The differences in the amplitude and the phase of $R_{T}(\phi)$ oscillations in the two cases indicate that the current flow through the area outside of the circle affects the measurements. Moreover, the current flow here is not strictly uni-directional. Thus, the shadowmask method is less direct than the sun-beam lithography method, which requires no modeling. But our key observations of angular oscillations are the same for both methods, ruling out artifacts due to lithographic processing. 

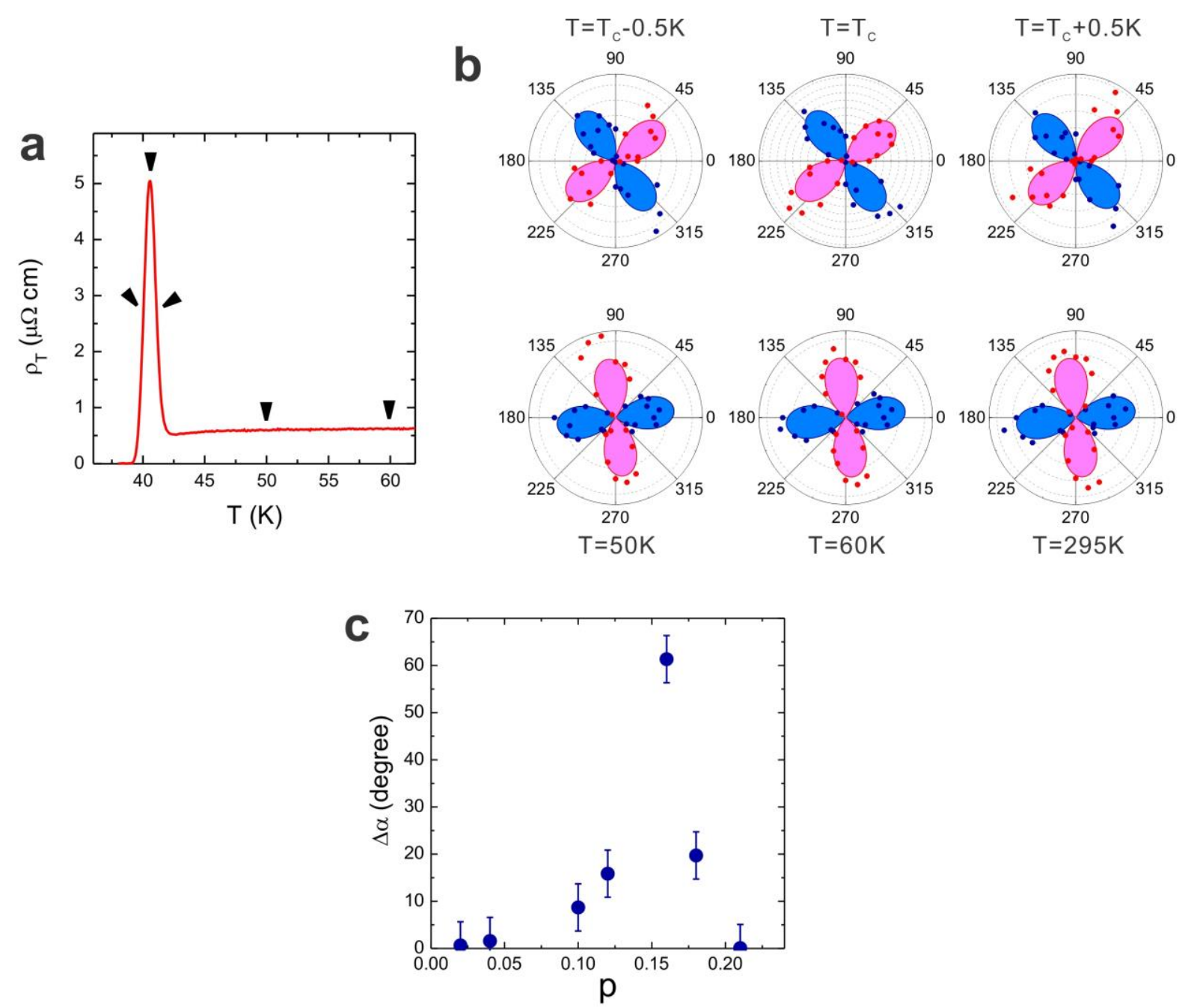

Extended Data Figure 8 | Rotation of $\alpha$, the direction of maximum longitudinal resistivity, with temperature. a, The $\rho_{T}\left(T, \phi=-30^{\circ}\right)$ data for an optimally doped $(p=0.16)$ LSCO showing at which temperature the measurements were made. $\mathbf{b}$, The polar plots of $\rho_{T}(\phi)$ at the temperatures as indicated. The radial distance measures the magnitude of $\rho_{T}$, with the positive values in blue and negative in red. (For better visibility, the radial extents here are all normalized to the same size.) Here and in the text $T_{c}$ refers to the midpoint of the resistive transition, which approximately coincides with the maximum of $\rho_{T}^{0}(T)$. Apparently, the nematic director of superconducting fluctuations has a different orientation from that in the 'normal' state at higher temperatures. c. Dependence of the difference $\Delta \alpha=\left|\alpha(T=295 \mathrm{~K})-\alpha\left(T=T_{\mathrm{c}}\right)\right|$ on doping. The angular resolution, and the upper limit on the error bar of $\alpha$, is $\pm 5^{\circ}$.

Note that in all samples $\alpha$ stays essentially constant from room temperature down to the onset of the superconducting transition, suggesting that the observed director 'rotation' may in fact result from different preferred (i.e., highest conductivity) directions in the normal state and in the region of superconducting fluctuations. 

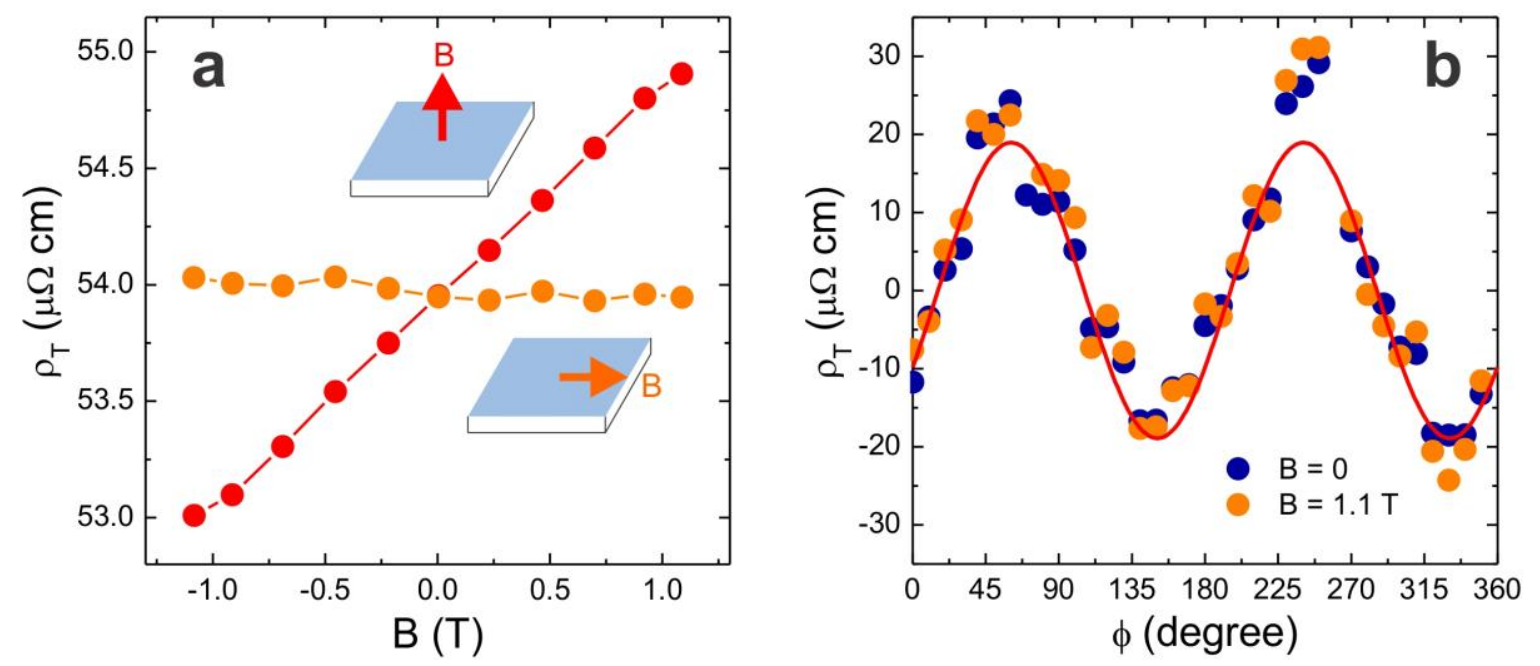

Extended Data Figure 9 | Resilience of the transverse resistivity in LSCO to the applied magnetic field. a, Orange solid dots: dependence of $\rho_{T}$ measured in one LSCO $(p=0.07)$ device at $T=295 \mathrm{~K}$, on the magnetic field applied parallel to the film surface. No significant effect is observed up to $B=1.1 \mathrm{~T}$. Red solid dots: transverse resistivity measured with the field perpendicular to the film surface, showing a pronounced Hall effect. Note, however, that it has an offset that is not $B$-dependent, i.e., the Hall signal is superposed on top of the 'nematic' one. $\mathbf{b}$, A detailed comparison of the $\rho_{T}(\phi)$ angular dependence measured using a sunbeam-patterned LSCO $(p=0.10)$ film at $T=$ $295 \mathrm{~K}$ shows that $B=1.1 \mathrm{~T}$ in-plane field affects neither the nematicity amplitude nor the director orientation. Blue solid dots: data at $B=0$; orange solid dots: data at $B=1.1 \mathrm{~T}$; red line: the fit. 

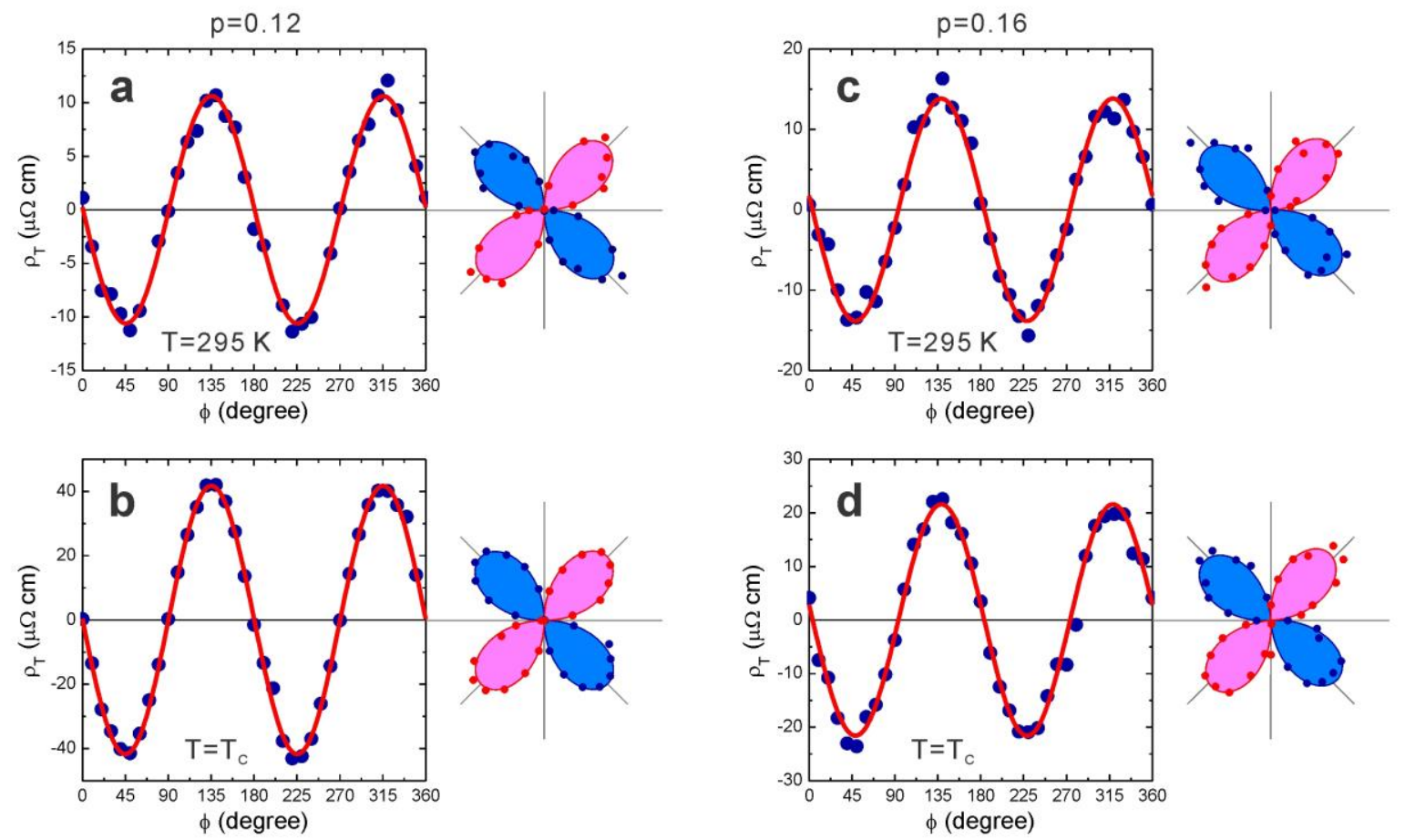

Extended Data Figure 10 | Angular dependence of $\rho_{T}$ in orthorhombic LSCO films. a, Blue dots: the $\rho_{T}(\phi)$ data, measured at $T=295 \mathrm{~K}$, in an underdoped $(p=0.12)$ LSCO film grown on, and epitaxially anchored to an orthorhombic $\mathrm{NdGaO}_{3}$ (NGO) substrate. The red lines are fits to $\rho_{T}(\phi)=\rho_{T}{ }^{0} \sin [2(\phi-\alpha)]$. b. The same, measured at $T_{c^{-}}$ midpoint. c, $\rho_{T}(\phi)$ at $T=295 \mathrm{~K}$ in an optimally doped $(p=0.16)$ LSCO film on NGO substrate. d, The same, measured at $T_{c}$-midpoint.

In these films, due to a significant ( 1\%) orthorhombic distortion, the nematic director is pinned to one of the crystal axes at all temperatures, i.e., it does not rotate with $p$ and $T$. 